\title{
Filogenia de avispas del género Netelia (Hymenoptera: Ichneumonidae) con énfasis en las especies costarricenses
}

\author{
James Coronado-Rivera ${ }^{1,2,3}$ \\ 1. Maestría en Biología, Sistema de Estudios de Posgrado, Universidad de Costa Rica, 11501-2060, San José, Costa \\ Rica; james_coronadorivera@yahoo.es \\ 2. Apartado 0823-01033, Panamá, República de Panamá. \\ 3. Dirección actual: Instituto Smithsonian de Investigaciones Tropicales de Panamá, Panamá.
}

\author{
Recibido 28-IX-2007. Corregido 18-V-2009. Aceptado 23-VII-2009.
}

\begin{abstract}
Phylogeny of wasps of the genus Netelia (Hymenoptera: Ichneumonidae) with emphasis on Costa Rican species. Results on the first cladistic analysis of the genus Netelia are presented. The analysis was carried out with the aid of the Hennig86 and Tree Gardener 2.2 programs, using a matrix of 79 taxa and 149 characters, based mainly on external morphology and male genitalia; only three characters are related to the natural history of the species. Within the in-group, representative species of the 11 subgenera of Netelia described in the world were included, although almost $80 \%$ of the species examined are from Costa Rica. To test the monophyly of the genus, five tryphonine species - Neliopisthus yui, Chiloplatys lucens, Phytodietus (Neuchorus) penai, P. (N.) lindus and $P$. (Phytodietus) moragai - were also included in the study group. Labena zerita (Labeninae) was used as an out-group. The search strategies of the trees included phylogenetic reconstruction by parsimony, without and with successive weighting of characters, through the commands bb *and mhennig*. In the analysis without successive weighting, 1211 equally parsimonious trees with a length of 649 steps, Ci of 23 and Ri of 68, were obtained. In the analysis with successive weighting 1211 trees with a length of 719 steps, Ci of 20 and $\mathrm{Ri}$ of 64, were obtained. The manifold cladograms obtained were summarized through two Majority Rule Consensuses trees, one for each rearch strategy. Support was found for the monophyly of the genus Netelia, and the subgenera N. (Apatagium), N. (Bessobates), N. (Monomacrodon), N. (Parabates), N. (Protonetelia), $N$. (Toxochilus) and $N$. (Longiterebates), although this last subgenus was only monophyletic in the analysis without successive weighting. Phylogenetic support was also obtained for the species groups leo, unguicularis and emorsa proposed by Townes (1938) in the subgenus $N$. (Netelia). The results support the synonymy of $N$. (Toxochiloides) with the group emorsa in the New World. Phylogenetic support was not found for the subgenera N. (Netelia), N. (Prosthodocis) and N. (Paropheltes), nor for the species groups virgata in N. (Bessobates) and geminata Townes in N. (Netelia). Nor was support found for a phylogenetic relation between the subgenera $N$. (Apatagium) and N. (Monomacrodon). Finally, with the results obtained here, the presence in Costa Rica of the subgenera $N$. (Longiterebates) and N. (Parabates), as documented by Gauld (1997), is rejected. The presence of N. (Bessobates) is unconditionally accepted and, for practical reasons, that of N. (Prosthodocis) and N. (Netelia); in addition, the presence of $N$. (Paropheltes) and $N$. (Toxochilus) is tentatively proposed. Rev. Biol. Trop. 57 (Suppl. 1): 213-238. Epub 2009 November 30.
\end{abstract}

Key words: phylogeny, morphology, male genitalias, parasitoid species, Tryphoninae, Netelia, insects, Costa Rica.

Netelia Gray es un género grande de avispas de la familia Ichneumonidae con 348 especies descritas en el mundo, las cuales habitan casi todas las regiones geográficas, exceptuando sólo las polares (Yu \& Horstmann 1997, Porter 1998, Kasparyan \& Tolkanitz 1999).
Townes (1938) en su revisión taxonómica de Netelia para el Neártico amplió la definición del género incluyendo en éste, junto a $N$. (Netelia) Gray 1860, a los subgéneros N. (Parabates) Förster 1869, N. (Paropheltes) Cameron 1907, N. (Apatagium) Enderlein 
1912, N. (Prosthodocis) Enderlein 1912, N. (Bessobates) Townes, Townes \& Gupta 1961 [= Parabatus Morley 1913], N. (Monomacrodon) Cushman 1934 y N. (Toxochilus) Townes 1938; los cuales, exceptuando a $N$. (Toxochilus), antes de Townes compartían la jerarquía taxonómica de géneros. Después de Townes (1938) tres subgéneros han sido descritos: $N$. (Toxochiloides) Tolkanitz 1974, N. (Longiterebates) Kaur \& Jonathan 1976 y N. (Protonetelia) Konishi 1986.

En Costa Rica muchas especies cumplen fielmente con las definiciones establecidas para subgéneros como $N$. (Bessobates), pero no así para otros documentados para el país por Gauld (1997), como N. (Parabates), N. (Prosthodocis) y N. (Longiterebates). Esto produce confusión en las determinaciones de los subgéneros a los que pertenecen las especies costarricenses, las cuales pueden ser más de 184 en el país (Coronado 2007). Por otro lado, la duda sobre la monofilia de algunos subgéneros, principalmente del mayor en número de especies $N$. (Netelia), ya ha sido planteada con anterioridad por Gauld (1984) y Konishi (2005). El presente trabajo tuvo como objetivo poner a prueba por primera vez la monofilia de los subgéneros de Netelia; además, reconocer cuáles son los que están presentes en Costa Rica.

\section{MATERIALES Y MÉTODOS}

Taxones: Setenta y tres de las 78 especies del grupo de estudio son del género Netelia, éstas representan a los 11 subgéneros descritos en el mundo y a la mayor variación morfológica observada entre las especies costarricenses. Casi el $80 \%$ de las especies de Netelia incluidas en el análisis son de Costa Rica.

Los subgéneros y los grupos a los que pertenecen las especies de Costa Rica fueron determinados a través de las claves ofrecidas por Townes (1938, 1969), Townes \& Townes (1966), Kaur \& Jonathan (1979) y Gauld (1997). Para efectos de esta investigación, son los que se encuentran en la lista de especies utilizadas para los análisis. Sin embargo, los desacuerdos en las identificaciones según la clave utilizada no fueron escasos. Las especies $N$. (Prosthodocis) sp. 1, sp. 3 hasta sp. 13, pueden ser determinadas como pertenecientes al subgénero $N$. (Longiterebates), mientras que especímenes atípicos (sin areoleta en las alas anteriores) de $N$. (Bessobates) pueden ser considerados como $N$. (Parabates) y la especie N. (Paropheltes) sp. 14 puede ser identificada como perteneciente al subgénero $N$. (Netelia) si se utiliza la clave de Gauld (1997) y no se examinan sus órganos genitales masculinos. Por otro lado, las especies N. (Netelia) sp. 29 y 30 pueden ser determinadas como N. (Prosthodocis), si sólo consideramos los caracteres de la morfología de los órganos genitales de los machos, y las especies $N$. (Netelia) sp. 51-53 pueden ser determinadas como $N$. (Toxochilus), si sólo consideramos los caracteres de su morfología externa, tal como fueron descritos por Townes (1938), y aceptados por Townes \& Townes (1966), Townes (1969) y Kaur \& Jonathan (1979).

Los especímenes costarricenses son parte del gran inventario de la fauna de himenópteros realizado por más de dos décadas y en la mayoría de los hábitats terrestres presentes en el país (Gámez \& Gauld 1993, Hanson \& Gauld 1995); las especies que representan serán formalmente descritas en trabajos alfataxonómicos posteriores.

Para seis de los 11 subgéneros descritos de Netelia en el Mundo fueron revisados especímenes representantes de sus especies tipos. Para el subgénero monotípico $N$. (Protonetelia) no se pudo revisar directamente ningún espécimen, en este caso se tomaron los datos a partir de las descripciones realizadas para el subgénero y la especie $N$. (Pt.) hirashimai por Konishi (1986).

Para poner a prueba la monofilia del género Netelia dentro del grupo de estudio también se incluyó cinco especies trifoninas, tres de éstas de su género hermano Phytodietus (Gauld 1997).

La especie utilizada como grupo externo Labena zerita (Labeninae) pertenece a una de las dos subfamilias de Ichneumonidae que se disputan la posición más basal dentro de esta familia de himenópteros (Quicke et al. 1999, Gauld \& Wahl 2000). 
El material biológico examinado pertenece a las siguientes instituciones: AEI: American Entomological Institute, Gainesville-FloridaEEUU; INBio: Instituto Nacional de Biodiversidad, Santo Domingo de Heredia-Costa Rica; IZPAN: Instytut Zoologiczny, Polska Akademia Nauk, ul Wilzca 64, Warszawa-Warsaw-Polonia; MZUCR: Museo de Zoología, Colección de Insectos, Escuela de Biología, Universidad de Costa Rica, San Pedro de Montes de Oca, San José-Costa Rica; y NHM: Natural History Museum, Londres-Inglaterra.

\section{Grupo externo:}

1. Labena zerita Gauld 2000: Labeninae; 1 T $10^{\lambda}$ determinados por J. Coronado-Rivera, Costa Rica MZUCR

\section{Grupo interno:}

2. Neliopisthus yui Gauld 1997: Tryphoninae, Oedemopsini; $\&$ Paratipo, $10^{\top}$ determinado por J. Coronado-Rivera, Costa Rica MZUCR

3. Chiloplatys lucens Townes y Townes 1945: Tryphoninae, Tryphonini; $10^{\top}$ determinado por J. Coronado-Rivera, Costa Rica MZUCR

4. Phytodietus (Neuchorus) lindus Gauld 1997: Tryphoninae, Phytodietini; $2 \sigma^{\top} \sigma^{\top}$ determinados por J. Coronado-Rivera, 19 determinada por Paul Hanson, Costa Rica MZUCR

5. P. (Neuchorus) penai Gauld 1997: Tryphoninae, Phytodietini; $q$ Paratipo, $1 \sigma^{\nearrow}$ determinado por J. Coronado-Rivera, Costa Rica MZUCR

6. P. (Phytodietus) moragai Gauld 1997: Tryphoninae, Phytodietini; $10^{\top}$ determinado por J. Coronado-Rivera, Costa Rica MZUCR

7. Netelia (Monomacrodon) bicolor (Cushman 1934): $0^{7}$ Paratipo 50150, especie tipo para el subgénero, Mt Omei, Szechuen, China AEI

8. N. (Apatagium) pagoni Gauld 1983: $20^{\top} \sigma^{\top}$ determinados por J. Coronado-Rivera, Brunei: BK. Retak, Brunei NHM
9. $\quad$ N. (Apatagium) retaki Gauld 1983: 2 \% determinadas por J. Coronado-Rivera, Papua-New Guinea NHM

10. N. (Apatagium) $n r$ retaki: 1 Q $1 O^{\top}$ determinados por J. Coronado-Rivera, Alcate Vict Mdro NHM

11. N. (Protonetelia) hirashimai Konishi 1986: Nueva Guinea

12. N. (Prosthodocis) antefurcalis (Szépligeti 1908): 1 \& $10^{\top}$ determinados por A. Bennett, especie tipo para el subgénero, $q$ : N - Rhodesia, O': Port. St. Johns, SO Afr., África AEI

13. N. (Prosthodocis) exserta (Cushman 1924): $1 \bigcirc 10^{\pi}$ determinados por $\mathrm{H}$. Tornes, $\mathrm{O}$ : Westerly, R.I. O': nr. Tiegerville, Norteamérica AEI

14. N. (Prosthodocis) sp. 1: 2 q Costa Rica INBio MZUCR NHM

15. N. (Prosthodocis) sp. 2: $3 \Varangle Q 10^{\top}$ Costa Rica INBio MZUCR NHM

16. N. (Prosthodocis) sp. 3: 4 $\bigcirc$ Costa Rica INBio MZUCR NHM

17. N. (Prosthodocis) sp. 4: $32 \bigcirc \bigcirc 8 \sigma^{\top} \sigma^{\top}$ Costa Rica INBio MZUCR NHM

18. N. (Prosthodocis) sp. 5: $3 \bigcirc Q 10 \circlearrowleft^{\top} \sigma^{\top}$ Costa Rica INBio MZUCR NHM

19. N. (Netelia) strigata (Enderlein 1912): $\sigma^{\top}$ Holotipo México IZPAN, $4 \sigma^{\top} \sigma^{\lambda}$ Costa Rica INBio MZUCR NHM

20. N. (Prosthodocis) sp. 6: 11 OQ $17 ठ^{\top} \sigma^{\nearrow}$ Costa Rica INBio MZUCR NHM

21. N. (Prosthodocis) sp. 7: $2 \sigma^{\top} \sigma^{\top}$ Costa Rica INBio MZUCR NHM

22. N. (Prosthodocis) sp. 8: $1 \sigma^{\pi}$ Costa Rica INBio MZUCR NHM

23. N. (Prosthodocis) sp. 9: 10 Costa Rica INBio MZUCR NHM

24. N. (Prosthodocis) sp. 10: 19 Costa Rica INBio MZUCR NHM

25. N. (Prosthodocis) sp. 11: 10 Costa Rica INBio MZUCR NHM

26. N. (Prosthodocis) sp. 12: 25 Q 4 $4 \sigma^{\top} \sigma^{\top}$ Costa Rica INBio MZUCR NHM

27. N. (Prosthodocis) sp. 13: 10 COSTA RICA INBio MZUCR NHM

28. $\quad N$. (Longiterebates) himalayensis Kaur y Jonathan 1976: 1 \% $10^{\top}$ Paratipos, especie 
tipo para el subgénero, H.P. - Dhekund, India AEI

29. N. (Longiterebates) turgida Kaur y Jonathan 1979: $1910^{\top}$ Paratipos, H.P. Kalatop (O), H.P. Shilaru $\left(O^{\top}\right)$, India AEI

30. N. (Paropheltes) barberi (Cushman 1924): $1 \uparrow 10^{\top}$ determinados por $\mathrm{H}$. Tornes, especie tipo para el grupo barberi, Mt. Madison, N.H., Norteamérica AEI

31. N. (Paropheltes) parvula (Meyer 1927): grupo tarsata, $1910^{\top}$ determinados por Vict Tolkanitz Rusia AEI

32. N. (Paropheltes) sp. 14: grupo radiata, 4 우 $50^{\top} \sigma^{\pi}$ Costa Rica INBio MZUCR NHM

33. N. (Toxochilus) clypeata (Cushman 1924): $1910^{\lambda}$ determinados por $\mathrm{H}$. Tornes, especie tipo para el subgénero, Q: $_{\text {: Elk Point, }}$

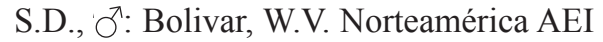

34. N. (Toxochilus) magniceps Townes 1939:

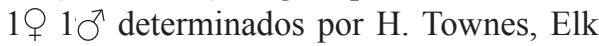
Point, S.D., Norteamérica AEI

35. N. (Parabates) nigricarpus (Thomson 1888): $10^{\pi} 59101$ determinado por J.F. Aubert, especie tipo para el subgénero, Alemania AEI

36. N. (Parabates) johnsoni (Ashmead 1900): 1 I $1 \mathrm{O}^{\top}$ determinados por A. Bennett Estados Unidos de Norteamérica AEI

37. N. (Bessobates) virgata (Geoffroy 1785): grupo virgata, $2 \sigma^{\top} \sigma^{\top}$ determinados por J.F. Perkins, Dartmoor and larix forest, Europa AEI

38. $\quad$ N. (Bessobates) sp. 15: grupo virgata, 1 q Costa Rica INBio MZUCR NHM

39. N. (Bessobates) sp. 16: grupo virgata, 2 $0^{\top} O^{\top}$ Costa Rica INBio MZUCR NHM

40. N. (Bessobates) sp. 17: grupo virgata, 1 + $30^{\top} \sigma^{\top}$ Costa Rica INBio MZUCR NHM

41. N. (Bessobates) sp. 18: grupo cristata, 2 OQ $10^{\top}$ Costa Rica INBio MZUCR NHM

42. N. (Bessobates) sp. 19: grupo virgata, 36 우 $320^{\top} \sigma^{\top}$ Costa Rica INBio MZUCR NHM

43. N. (Toxochiloides) latro Holmgren 1968: $191 O^{\pi}$ determinados por R. Kaur, $q$ : Assam: Khasi Hills, Cherrapunji, O`: Ulithi Atoll, Mog mog, Asia AEI
44. N. (Netelia) sp. 20: grupo geminata, 140 \% $110^{\top} O^{\top}$ Costa Rica INBio MZUCR NHM

45. N. (Netelia) sp. 21: grupo geminata, 11 q $23 \sigma^{\top} \sigma^{\top}$ Costa Rica INBio MZUCR NHM

46. N. (Netelia) tinctipennis (Cameron 1886): grupo geminata, $q$ Holotipo Panamá NHM, 9 OOQ $20^{\pi} O^{x}$ Costa Rica INBio MZUCR NHM

47. N. (Netelia) sp. 22: grupo geminata, 11 q $120^{\top} \sigma^{\top}$ Costa Rica INBio MZUCR NHM

48. N. (Netelia) sp. 23: grupo geminata, 5 Q $5 \sigma^{\top} \sigma^{\top}$ Costa Rica INBio MZUCR NHM

49. N. (Netelia) sp. 24: grupo geminata, $20^{\top} \sigma^{\pi}$ Costa Rica INBio MZUCR NHM

50. N. (Netelia) sp. 25: grupo geminata, 6 우 $4 O^{\top} O^{\top}$ Costa Rica INBio MZUCR NHM

51. N. (Netelia) sp. 26: grupo geminata, 3 qQ Costa Rica INBio MZUCR NHM

52. N. (Netelia) sp. 27: grupo geminata, 31 \&O $6 \sigma^{\top} \sigma^{\top}$ Costa Rica INBio MZUCR NHM

53. N. (Netelia) sp. 28: grupo geminata, 1 q Costa Rica INBio MZUCR NHM

54. N. (Netelia) sp. 29: grupo geminata, $10^{\top}$ Costa Rica INBio MZUCR NHM

55. N. (Netelia) sp. 30: grupo geminata, $10^{\top}$ Costa Rica INBio MZUCR NHM

56. N. (Netelia) sp. 31: grupo emorsa, 7 Q $110^{\top} \sigma^{\pi}$ Costa Rica INBio MZUCR NHM

57. N. (Netelia) sp. 32: grupo emorsa, 2 우 $40^{\lambda} \sigma^{\top}$ Costa Rica INBio MZUCR NHM

58. N. (Netelia) sp. 33: grupo leo, 7 Q $\bigcirc$ Costa Rica INBio MZUCR NHM

59. N. (Netelia) sp. 34: grupo leo, 45 우 $10^{\nearrow}$ Costa Rica INBio MZUCR NHM

60. N. (Netelia) sp. 35: grupo geminata, $4 \sigma^{\top} \sigma^{\top}$ Costa Rica INBio MZUCR NHM

61. N. (Netelia) sp. 36: grupo geminata, 7 q $100^{\top} \sigma^{\top}$ Costa Rica INBio MZUCR NHM

62. N. (Netelia) sp. 37: grupo geminata, 10 \&O $60^{\top} \sigma^{\top}$ Costa Rica INBio MZUCR NHM

63. N. (Netelia) sp. 38: grupo geminata, $13 \sigma^{\top} \sigma^{\top}$ Costa Rica INBio MZUCR NHM

64. N. (Netelia) sp. 39: grupo geminata, $10^{\top}$ Costa Rica INBio MZUCR NHM

65. N. (Netelia) sp. 40: grupo geminata, $20^{\top} \sigma^{\top}$ Costa Rica INBio MZUCR NHM

66. N. (Netelia) sp. 41: grupo geminata, $100^{\top} \sigma^{\top}$ Costa Rica INBio MZUCR NHM 
67. N. (Netelia) sp. 42: grupo geminata, $10^{\pi}$ Costa Rica INBio MZUCR NHM

68. N. (Netelia) sp. 43: grupo geminata, 18 ㅇ $24 \sigma^{\top} \sigma^{\top}$ Costa Rica INBio MZUCR NHM

69. N. (Netelia) sp. 44: grupo unguicularis, $10^{\top}$ Costa Rica INBio MZUCR NHM

70. N. (Netelia) sp. 45: grupo unguicularis, 7 7O $230^{\top} \sigma^{\top}$ Costa Rica INBio MZUCR NHM

71. N. (Netelia) sp. 46: grupo unguicularis, 13 우 $290^{\top} \sigma^{\top}$ Costa Rica INBio MZUCR NHM

72. N. (Netelia) ignota (Morley 1913): grupo unguicularis, Ơ Holotipo Panamá NHM, 20 Oᄋ $340^{\top} \sigma^{\top}$ Costa Rica INBio MZUCR NHM

73. N. (Netelia) sp. 47: grupo unguicularis, $10^{\top}$ Costa Rica INBio MZUCR NHM

74. N. (Netelia) sp. 48: grupo unguicularis, $3 \bigcirc 0$ 1 $10^{\top}$ Costa Rica INBio MZUCR NHM

75. N. (Netelia) sp. 49: grupo unguicularis, 23 우 $160^{\top} \sigma^{\top}$ Costa Rica INBio MZUCR NHM

76. N. (Netelia) sp. 50: grupo unguicularis, 14우 $40^{\top} \sigma^{\top}$ Costa Rica INBio MZUCR NHM

77. N. (Netelia) sp. 51: grupo geminata, 1 \& 3 $0^{\top} \sigma^{\top}$ Costa Rica INBio MZUCR NHM

78. N. (Netelia) sp. 52: grupo geminata, 14 우 $320^{\top} \sigma^{\top}$ Costa Rica INBio MZUCR NHM

79. N. (Netelia) sp. 53: grupo geminata, $4 \sigma^{\top} \sigma^{\top}$ Costa Rica INBio MZUCR NHM

Caracteres: Los caracteres son los estados apomórficos de atributos multiestados; excepto por el número 80 , todos son binarios y se omitió la descripción de los estados plesiomórficos. La presencia del carácter es codificada en la matriz como 1 y la ausencia como 0 .

El $72 \%$ de los caracteres son descritos de la morfología externa de ambos sexos y de los órganos reproductores externos de las hembras, el $26 \%$ se describen de los órganos genitales de los machos y el $2 \%$ de la biología, la morfología de los huevos y la cápsula cefálica de las larvas. La terminología se ilustra en las Fig. 1-5 y algunos caracteres en las Fig. 6-65.

\section{Descripción de los caracteres}

\section{Relacionados con la biología \\ de las especies:}

1. Durante la oviposición el huevo es sujetado externamente al hospedero por medio de un pedúnculo o ancla que es insertado en la cutícula con la ayuda del ovipositor (Gauld 1997).

2. Huevos con pedúnculo alargado y en espiral, sin ancla (Gauld 1997).

3. Ultimo estadio larval con plato esclerosado desde el labio ventral hasta el esclerito labial (Short 1978, Gauld 1997).

\section{Morfología externa:}

4. Parte frontal de la cabeza sin pigmentación oscura negra o parda.

5. Parte posterior de la cabeza sin pigmentación oscura negra o parda.

6. Pronoto sin pigmentación oscura negra o parda.

7. Mesoescudo sin pigmentación oscura negra o parda.

8. Escutelo sin pigmentación oscura negra o parda.

9. Mesopleura sin pigmentación oscura negra o parda.

10. Metapleura sin pigmentación oscura negra o parda.

11. Propodeo sin pigmentación oscura negra o parda.

12. T1 sin pigmentación oscura negra o parda.

13. T2 sin pigmentación oscura negra o parda.

14. T3 sin pigmentación oscura negra o parda.

15. T4 sin pigmentación oscura negra o parda.

16. T5 sin pigmentación oscura negra o parda.

17. T6 sin pigmentación oscura negra o parda.

18. T7 sin pigmentación oscura negra o parda. 


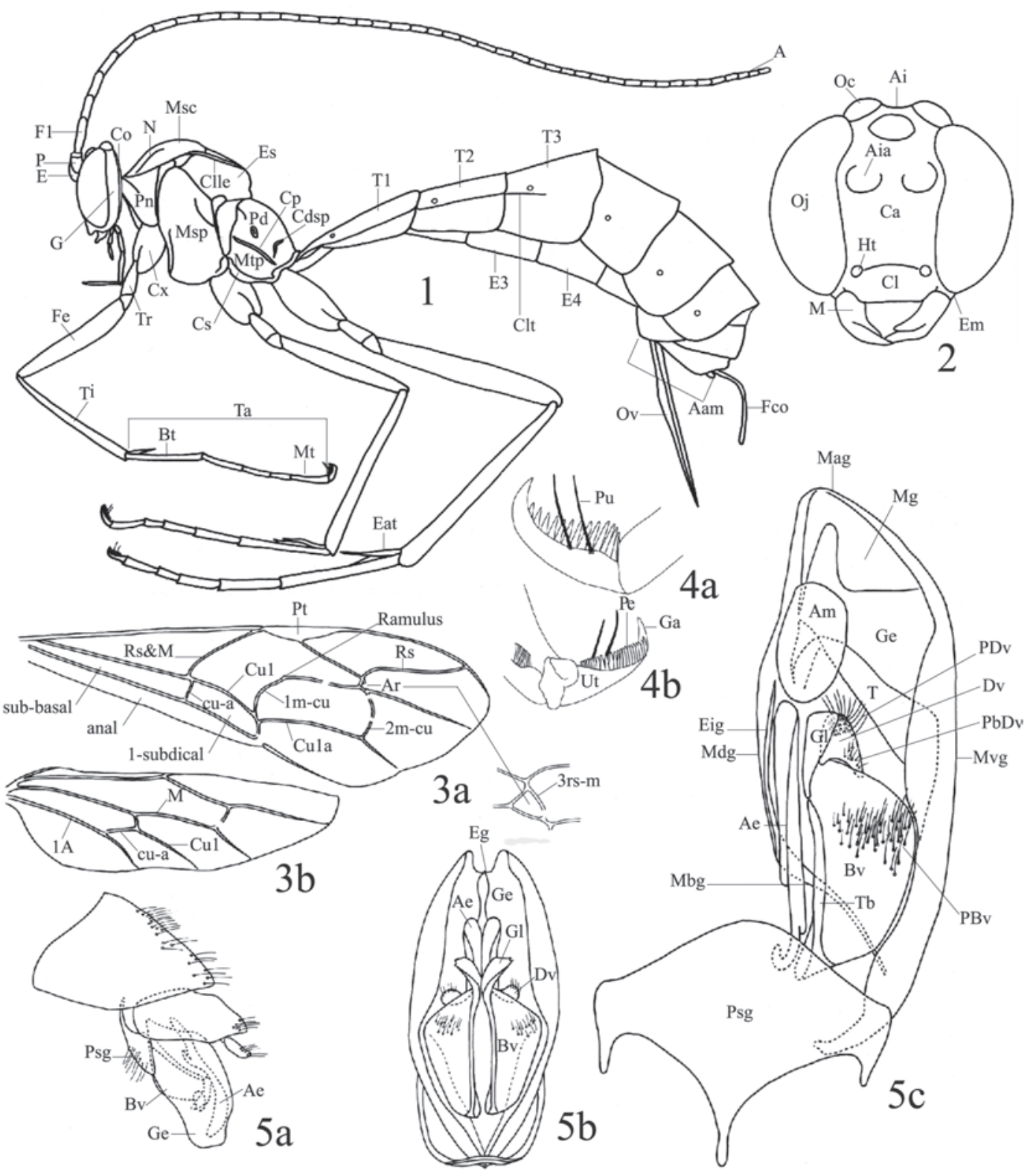

Fig. 1-5. Netelia generalizado, rotulado para mostrar terminología. 1: cuerpo completo, vista lateral; 2: cabeza, vista frontal; 3a: ala anterior; 3b: ala posterior; 4a: uña tarsal anterior, hembra; 4b: uña tarsal anterior, macho; 5a: genitales de macho, vista lateral; 5b: genitales de macho, vista ventral; 5c: valva derecha de los genitales de macho, vista interior.

$\mathbf{A}=$ antena; $\mathbf{A} \mathbf{a m}=$ altura apical del metasoma; $\mathbf{A e}=$ aedeago; $\mathbf{A} \mathbf{i}=$ área interocelar; $\mathbf{A} \mathbf{i a}=$ área de inserción antenal; $\mathbf{A m}=$ almohadilla; $\mathbf{A r}=$ areoleta; $\mathbf{B t}=$ basitarsómero; $\mathbf{B v}=$ basivolsella; $\mathbf{C a}=$ cara; $\mathbf{C d s p}=$ cresta dorsal subapical del propodeo; $\mathbf{C l}=$ clípeo; Clle= carena longitudinal lateral del escutelo; $\mathbf{C l t}=$ carena del lateroterguito del segmento 3; $\mathbf{C o}=$ carena occipital; $\mathbf{C p}=$ carena pleural; $\mathbf{C s}=$ carena submetapleural; $\mathbf{C x}=$ coxa; $\mathbf{D v}=$ distiovolsella; $\mathbf{E}=$ escapo; $\mathbf{E a t}=$ espolón apical de la tibia; $\mathbf{E i g}=$ escleroma interno de la gonoescama; $\mathbf{E g}=$ espina apical de la gonoescama; $\mathbf{E m}=$ espacio malar; $\mathbf{E s}=$ escutelo; $\mathbf{E 1}-\mathbf{E 8}=$ esternos 1 al 8; $\mathbf{F} \mathbf{c o}=$ fundas cobertora del ovipositor; $\mathbf{F e}=$ fémur; $\mathbf{F 1}=$ flagelo antenal: primer flagelómero; $\mathbf{G}=$ gena; $\mathbf{G a}=$ garfio; $\mathbf{G e}=$ gonoescama; $\mathbf{G l}=$ gonolacinia; $\mathbf{H t}=$ hoyo tentorial; $\mathbf{M}=$ mandíbulas; $\mathbf{M a g}=$ margen apical de la gonoescama; $\mathbf{M b g}=$ margen basal de la gonoescama; $\mathbf{M d g}=$ margen dorsal de la gonoescama; $\mathbf{M g}=$ membrana de la gonoescama; $\mathbf{M s c}=$ 
19. T8 sin pigmentación oscura negra o parda.

20. E3 sin pigmentación oscura negra o parda.

21. E4 sin pigmentación oscura negra o parda.

22. E5 sin pigmentación oscura negra o parda.

23. Mesoescudo con tres bandas de color más oscuro (puede ser ligeramente más oscuro) que el resto del mesosoma: negro o pardo oscuro o claro (Fig. 7, 9, compare con la Fig. 8 en donde el mesoescudo es completamente negro).

24. Patrón de coloración vespiforme: amarillo brillante con bandas negras (Fig. 6-7).

25. Flagelos antenales completamente sin pigmentación oscura (se ven del mismo color que el resto del cuerpo).

26. Área interocelar sin pigmentación oscura negra o parda.

27. Pterostigma sin pigmentación oscura negra o parda.

28. Mesosoma con marcas amarillas.

29. Relación altura de la cabeza / altura mayor del mesosoma $0.7 x$ o menos.

30. Relación altura de la cabeza / altura mayor del mesosoma $0.6 x$ o menos.

31. Relación altura de la cabeza / altura mayor del mesosoma $0.5 x$ o menos.

32. Clípeo pequeño, separado del borde interno de los ojos por $0.5 \mathrm{x}$ o más su ancho, hundido en su centro (Fig. 11a).

33. Clípeo grande unido o casi unido al borde interno de los ojos, cuando está separado de los ojos es por $0.1 \mathrm{x}$ o menos su ancho (Fig. 11b).

34. Clípeo corto, con el margen apical ampliamente cóncavo (Fig. 13-14).
35. Clípeo alargado, convexo, margen apical muy convexo sobrepasa el margen inferior de los ojos.

36. Mandíbulas torcidas: el diente superior es más grande y en posición anterior que el inferior (Fig. 11b-14).

37. Mandíbulas con diente superior muy grueso y con el ápice romo (Fig. 13-14).

38. Ojos muy grandes con una muesca en el margen interno justo a la altura de las inserciones antenales (Fig. 12-14).

39. Ocelos grandes: el anterior ocupa $0.4 \mathrm{x}$ o más del espacio entre los ojos; los posteriores pueden estar unidos o no al borde interno superior de los ojos, cuando separados sólo por $0.3 \mathrm{x}$ o menos sus diámetros.

40. Ocelos posteriores separados del borde superior de los ojos por $0.3 \mathrm{x}$ o menos sus diámetros.

41. Ocelos posteriores totalmente unidos al borde superior de los ojos.

42. Carena frontal presente: carena pequeña que se extiende desde el borde superior de los ojos hasta el margen externo de las inserciones de las antenas (Fig. 14).

43. Parte superior de la gena con una concavidad cerca de los bordes externos de los ocelos posteriores, en perfil el área interocelar se ve ligeramente elevada sobre el borde superior de los ojos (Fig. 12, 14).

44. Gena angosta: 0.9x o menos el ancho basal de las mandíbulas.

45. Gena muy angosta: $0.6 x$ o menos el ancho basal de las mandíbulas.

46. Carena occipital incompleta, ausente dorsalmente.

47. Carena occipital muy débil.

48. Carena occipital totalmente ausente.

mesoescudo; $\mathbf{M s p}=$ mesopleura; $\mathbf{M t}=$ metatarsómero; $\mathbf{M t p}=$ metapleura; $\mathbf{M v g}=$ margen ventral de la gonoescama; $\mathbf{N}=$ notauli; $\mathbf{O c}=$ ocelo; $\mathbf{O j}=$ ojo; $\mathbf{O v}=$ ovipositor; $\mathbf{P}=$ pedicelo; $\mathbf{P b D v}=$ pelos cortos basales de la distiovolsella; $\mathbf{P B v}=$ pelos de la basivolsella; $\mathbf{P d}=$ propodeo; $\mathbf{P D v}=$ pelos largos marginales de la distiovolsella; $\mathbf{P e}=$ peine; $\mathbf{P n}=$ pronoto; $\mathbf{P s g}=$ plato subgenital; $\mathbf{P t}=$ pterostigma; $\mathbf{P u}=$ pelos unguales; $\mathbf{T}=$ tirante; $\mathbf{T a}=$ tarsos: tarsómeros; $\mathbf{T} \mathbf{b}=$ tirante de la basivolsella; $\mathbf{T} \mathbf{i}=$ tibia; $\mathbf{T r}=$ trocanter; $\mathbf{T} 1-\mathbf{T} 9=\operatorname{tergos} 1$ al $9 ; \mathbf{U t}=$ uña tarsal. 


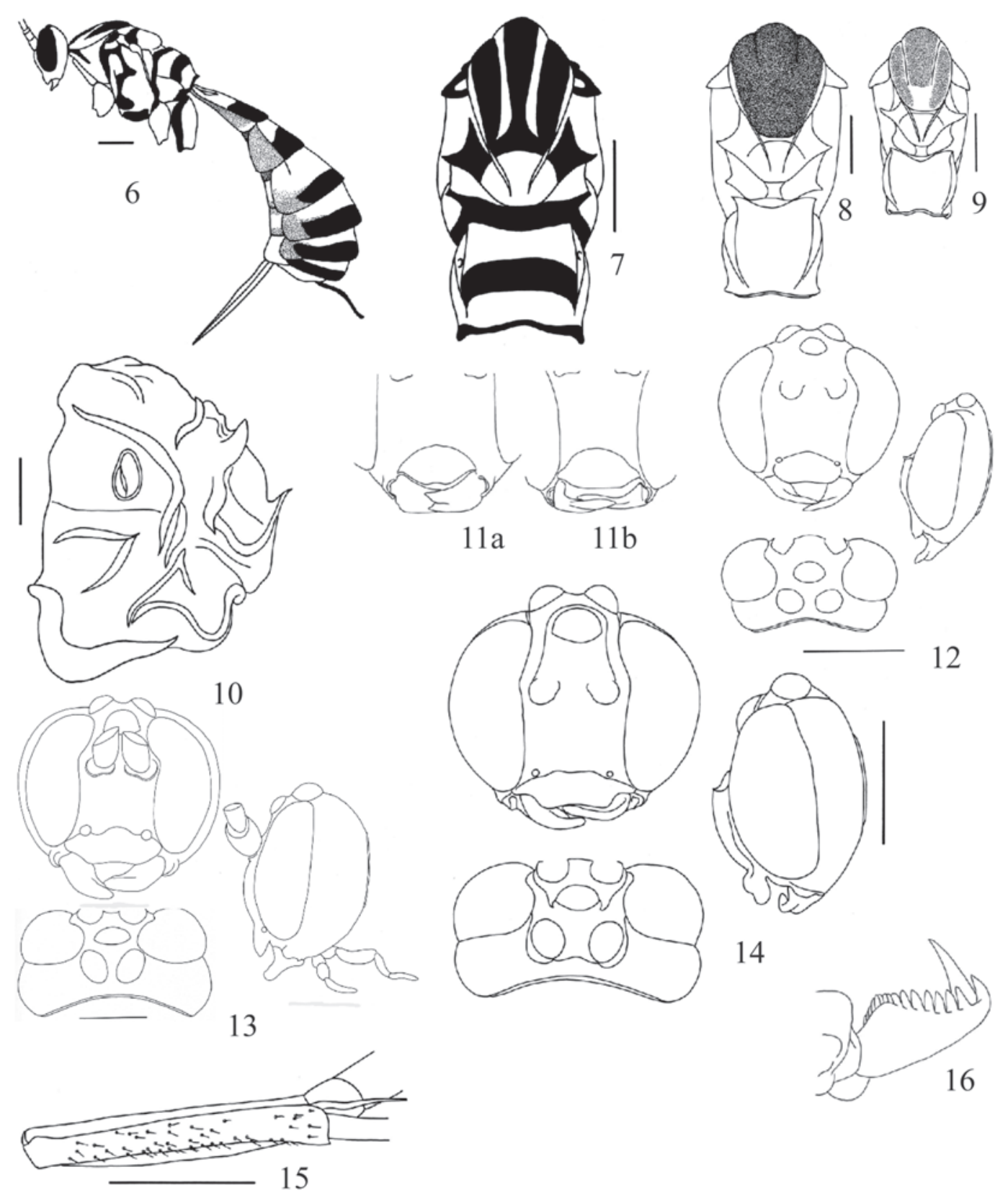

Fig. 6-16: Morfología externa: 6-7: N. (Prosthodocis) sp.2; 8: N. (Prosthodocis) sp.4; 9: N. (Netelia) strigata; 10: N. (Netelia) sp.49; 11a: Phytodietus; 11b: Netelia; 12: N. (Netelia) sp.28; 13: N. (Netelia) sp.51; 14-15: N. (Toxochilus) clypeata; 16: N. (Apatagium) sp. (Escala: $1.0 \mathrm{~mm})$.

Fig. 6-16: External morphology: 6-7: N. (Prosthodocis) sp.2; 8: N. (Prosthodocis) sp.4; 9: N. (Netelia) strigata; 10: N. (Netelia) sp.49; 11a: Phytodietus; 11b: Netelia; 12: N. (Netelia) sp.28; 13: N. (Netelia) sp.51; 14-15: N. (Toxochilus) clypeata; 16: N. (Apatagium) sp. (Scale: $1.0 \mathrm{~mm})$. 
49. Mesopleura mate (nunca pulida) con punturas conspicuas.

50. Mesopleura con punturas densas y pronunciadas (se ve granulada).

51. Notauli ausente o inconspicuo.

52. Carenas longitudinales laterales del escutelo conspicuas y completas.

53. Propodeo sin carenas (Gauld 1997).

54. Propodeo robusto, visto dorsalmente su largo 0.9x o menos su ancho basal.

55. Superficie dorsal del propodeo, antes de la cresta dorsal subapical, con estrías transversales conspicuas.

56. Superficie dorsal del propodeo, antes de la cresta dorsal subapical, con densas punturas pronunciadas y estrías muy finas (se ve granulado-estriolado).

57. Superficie dorsal del propodeo, después de la cresta dorsal subapical, con densas punturas pronunciadas (se ve granulado).

58. Cresta dorsal subapical del propodeo conspicua.

59. Cresta dorsal subapical del propodeo muy desarrollada y engrosada.

60. Metapleura conspicuamente puntuada.

61. Metapleura conspicua y densamente puntuada (se ve granulada).

62. Metapleura conspicuamente estriada.

63. Metapleura fuertemente estriada.

64. Carena submetapleural muy amplia basalmente (Fig. 10).

65. Carena pleural ausente o inconspicua.

66. Metasoma comprimido lateralmente.

67. Largo del tergo del primer segmento del metasoma 4.0x o más su ancho posterior (Fig. 25, compare con la Fig. 24).

68. Ovipositor: 3.0x la altura apical del metasoma, ápice del ovipositor con nudo subapical en las valvas superior e inferior, su ápice se ve lanceolado (Fig. 29).

69. Ovipositor: 2.0-1.0x la altura apical del metasoma, ápice agudo, sin rastro de dentición en la valva inferior.

70. Ovipositor: 2.0x la altura apical del metasoma, valva inferior con un nudo conspicuo en el centro de su largo (Fig. 28).

71. Ovipositor: 2.0x la altura apical del metasoma, valva inferior sin un nudo conspicuo en el centro de su largo.
72. Ovipositor corto: 1.0x la altura apical del metasoma, nunca sobrepasa el ápice del metasoma (Fig. 26-27).

73. Tibias anteriores con espolón apical alargado, el peine únicamente llega hasta su centro (Gauld 1997).

74. Tibias con pelos conspicuos, cortos, gruesos y numerosos en forma de espinas (Gauld 1997).

75. Pelos en forma de espinas de las tibias anteriores numerosos, las inserciones de la mayoría de ellos se encuentran separadas por menos que sus largos (Fig. 15).

76. Patas y antenas relativamente largas y delgadas.

77. Tarsómeros del primer par de patas en las hembras tubulares, con pocos pelos en la superficie dorsal y con la superficie ventral aterciopelada.

78. Uñas tarsales del primer par de patas en ambos sexos desde medianas hasta muy largas: su largo 5.5x o más su ancho basal.

79. Uñas tarsales del primer par de patas en ambos sexos muy largas: $0.7 x$ o más la distancia entre los hoyos tentoriales.

80. Peines en las uñas tarsales: ausentes (0); presentes (1); reducidos (2).

81. Peine de las uñas tarsales del último par de patas en ambos sexos sobrepasa el garfio de las uñas (Fig. 16).

82. Dientes, principalmente los centrales, de los peines de las uñas tarsales en las hembras relativamente cortos y muy anchos en sus bases.

83. Dientes de los peines de las uñas tarsales anteriores en los machos numerosos y muy juntos entre sí, no se ve luz entre ellos, principalmente entre los dientes centrales.

84. Garfio de las uñas tarsales posteriores abierto en ambos sexos, en ángulo mucho mayor que $90^{\circ}$, además el ápice del garfio sobrepasa por poco el ápice de los dientes del peine de la uña.

85. Uñas tarsales anteriores en los machos con dos o más pelos unguales.

86. Uñas tarsales anteriores en los machos con tres o más pelos unguales. 

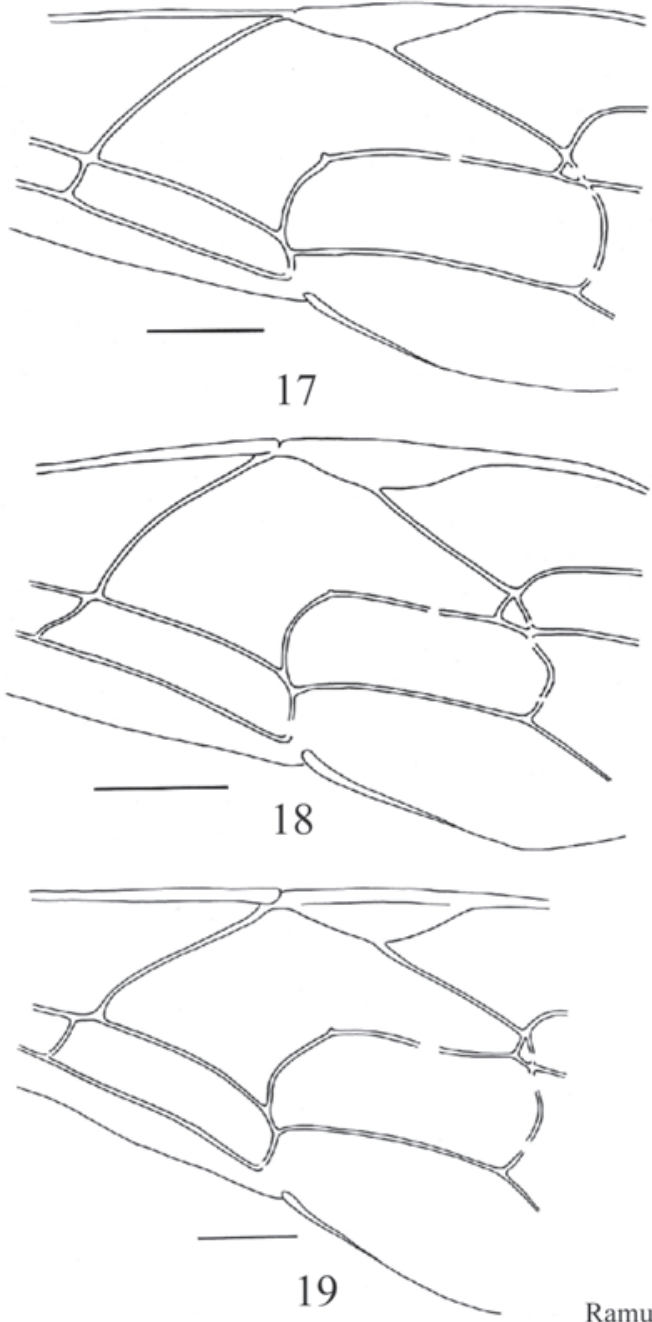

Ramulus

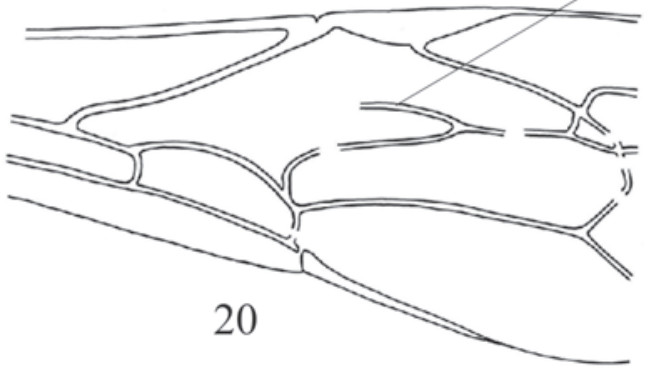

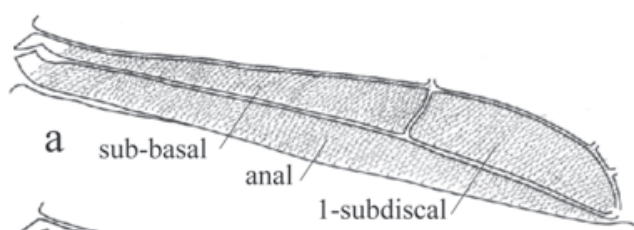
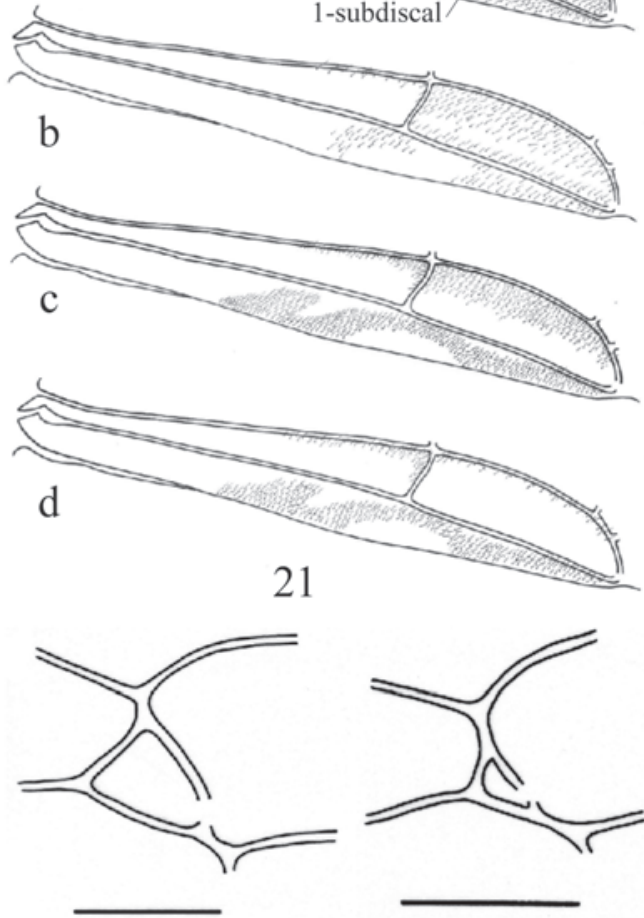

22

Fig. 17-23: Morfología externa: 17: N. (Prosthodocis) sp.2; 18: N. (Prosthodocis) sp.12; 19: N. (Prosthodocis) antefurcalis; 20: N. (Netelia) sp.; 21: a: Phytodietus; b-d: Netelia spp.; 22-23: N. (Bessobates) spp. (Escala: 1.0mm).

Fig. 17-23: External morphology: 17: N. (Prosthodocis) sp.2; 18: N. (Prosthodocis) sp.12; 19: N. (Prosthodocis) antefurcalis; 20: N. (Netelia) sp.; 21: a: Phytodietus; b-d: Netelia spp.; 22-23: N. (Bessobates) spp. (Scale: 1.0mm). 
87. Largo del primer par de alas superior a $11 \mathrm{~mm}$.

88. Largo del primer par de alas superior a $15 \mathrm{~mm}$.

89. Celda sub-basal sólo con un pequeño grupo de pelos próximos a sus márgenes superior y distal (Fig. 21b-d).

90. Celda sub-basal completamente sin pelos.

91. Celda 1-subdiscal con muy pocos pelos (Fig. 21b-d).

92. Celda 1-subdiscal sin pelos en la periferia de su margen inferior (Fig. 21c-d).

93. Celda anal con pocos pelos en un patrón en forma de arco (Fig. 21b-d).

94. Unión de la vena $1 m-c u$ en la vena $C u l$ próxima a la unión de la vena Cula por 8.0x o menos su ancho.

95. Vena $3 r s-m$ en las alas anteriores ausente.

96. Areoleta de las alas anteriores triangular: subsésil o peciolada.

97. Areoleta de las alas anteriores peciolada (Fig. 22-23).

98. Vena $c u-a$ distal a la base de la vena $R s \& M$ por 3.0x o más su ancho (Fig. 20)

99. Vena $\mathrm{cu}-a$ distal a la base de la vena $R s \& M$ por 7.0x o más su ancho.

100. Vena $c u-a$ proximal a la base de la vena $R s \& M$ por $7.0 \mathrm{x}$ o menos su ancho (Fig. 18-19).

101. Vena $c u-a$ curva.

102. Vena $c u$ - $a$ ligeramente quebrada arriba, a veces se ve sigmoidea (Fig. 18).

103. Vena $\mathrm{cu}-a$ ligeramente reclinada (Fig. 18).

104. Vena $2 m-c u$ arqueada (Fig. 17).

105. Vena $2 m-c u$ no inclinada.

106. Vena $2 m-c u$ muy arqueada.

107. Vena $2 m-c u$ recta, quebrada en su extremo superior y reclinada (Fig. 20).

108. Vena $R s \& M$ reclinada en ángulo entre $35^{\circ}$ y $50^{\circ}$ (Fig. 20).

109. Alas anteriores: vena ramulus presente (Fig. 20).

110. Vena Rs sigmoidea.

111. Alas posteriores: vena $C u 1$ se une a la vena $c u$ - $a$ más próxima de la vena $M$ que de la vena $1 A$.

\section{Morfología de los órganos genitales de los machos:}

112. Forma de la gonoescama nunca subtriangular, con el margen basal relativamente angosto $0.3-0.7 \mathrm{x}$ su largo (comparar Fig. 30 y 33 , con 34-37).

113. Gonoescama en forma de espátula, con el margen dorsal ampliamente cóncavo y el apical amplio y convexo (Fig. 35, 38, 47).

114. Gonoescama relativamente corta y ancha (Fig. 57-58, 64-65).

115. Escleroma interno paralelo al margen dorsal presente.

116. Escleroma interno paralelo al margen dorsal corto y muy grueso (Fig. 50, 57-58).

117. Espina pequeña conformada por una extensión de la gonoescama ubicada hacia el centro del borde dorsal de ésta.

118. Espina muy grande conformada por una extensión de la gonoescama ubicada hacia el ápice de ésta (Fig. 64-65).

119. Membrana que cubre la superficie interna de la gonoescama con algún tipo de modificación, ésta puede ser desde una ligera sombra, apenas perceptible al microscopio de luz, hasta una serie de estructuras especializadas como lo son la "almohadilla" y el "tirante" (todas las figuras de los órganos genitales de los machos, excepto 30 , 33, 47, 53-56).

120. Margen anterior de la membrana modificada que cubre la superficie interna de la gonoescama oblicuo, visible desde la esquina basal-dorsal hasta el medio del margen ventral de la gonoescama (Fig. 35, $38)$.

121. "Tirante" amplio y poco conspicuo su margen anterior es oblicuo desde el margen ventral hasta el apical de la gonoescama (Fig. 34, 37, 39).

122. "Tirante" amplio y poco conspicuo con el margen anterior bien definido (Fig. 39).

123. Tirante angosto, oblicuo o transversal, generalmente conspicuo, aunque a veces es vestigial, siempre inicia en el centro del 

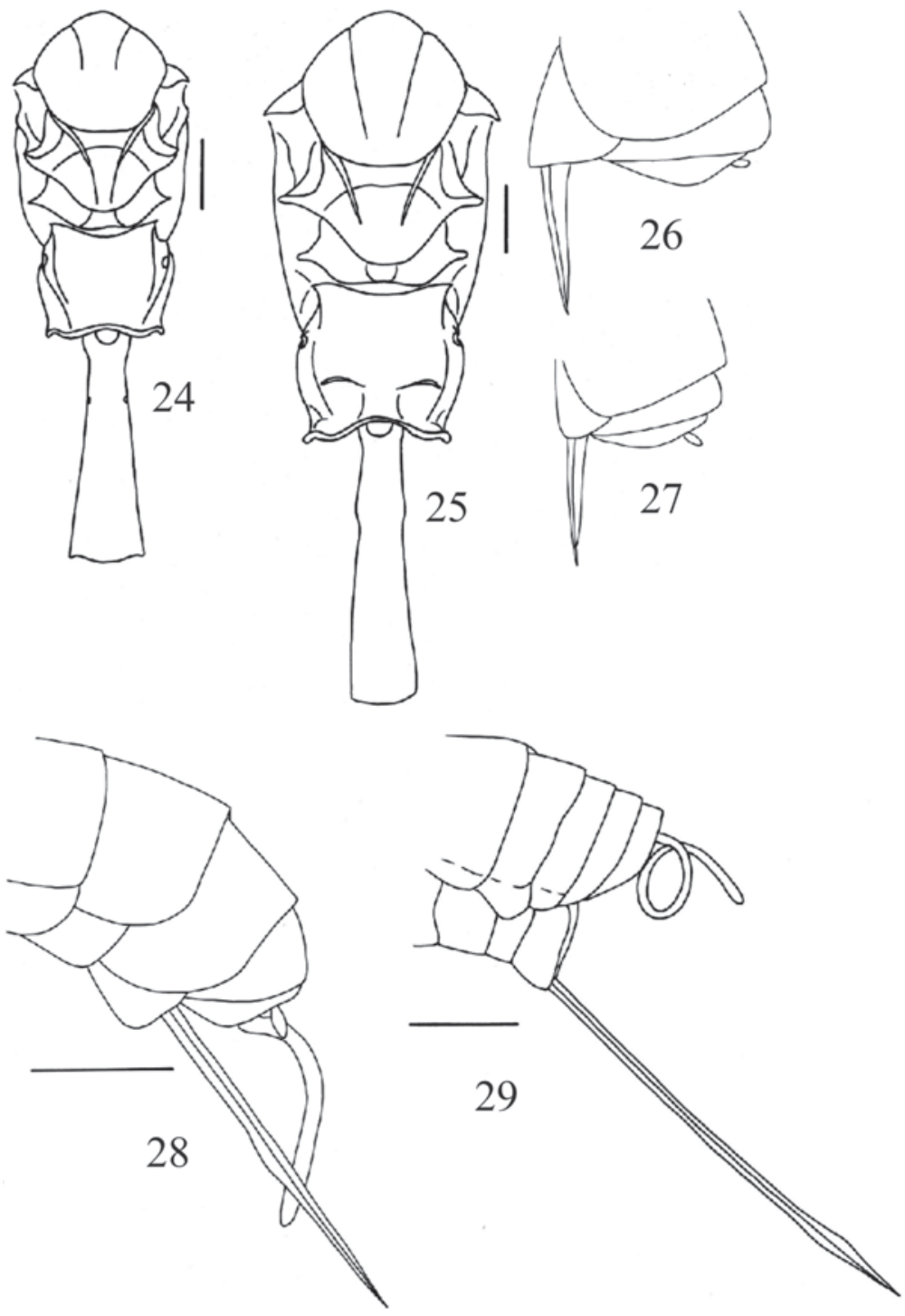

Fig. 24-29: Morfología externa: 24: N. (Bessobates) sp.19; 25: N. (Prosthodocis) sp;; 26-27: N. (Bessobates) spp.; 28: N. (Paropheltes) barberi; 29: P. (Neuchorus) lindus (Escala: 1.0mm).

Fig. 24-29: External morphology: 24: N. (Bessobates) sp.19; 25: N. (Prosthodocis) sp.; 26-27: N. (Bessobates) spp.; 28: N. (Paropheltes) barberi; 29: P. (Neuchorus) lindus (Scale: 1.0mm). 


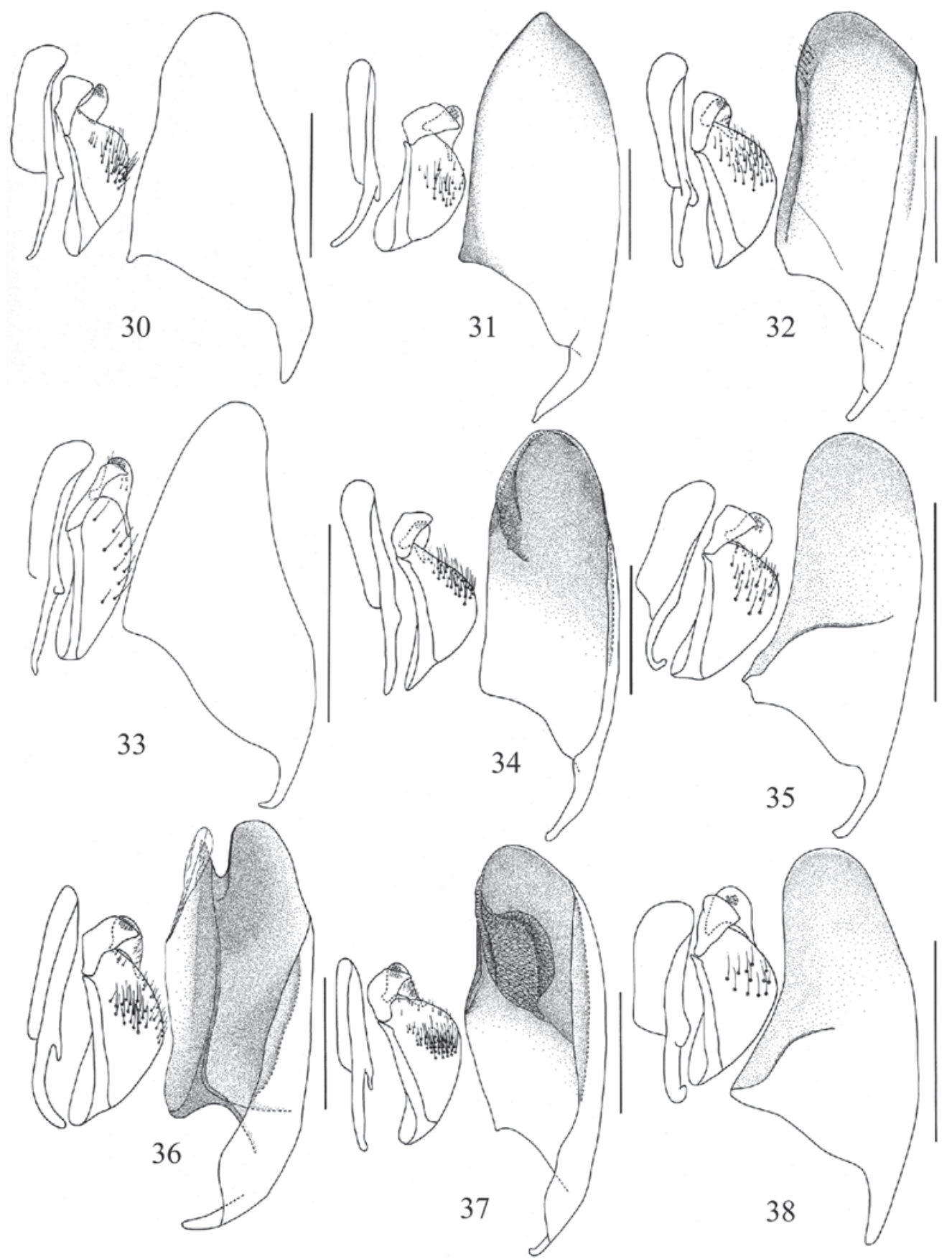

Fig. 30-38: Genitales de machos: 30: N. (Prosthodocis) sp.2; 31: N. (Prosthodocis) sp.; 32: N. (Prosthodocis) sp.7; 33: P. (Phytodietus) moragai; 34: N. (Prosthodocis) sp.6; 35: N. (Prosthodocis) sp.12; 36: N. (Prosthodocis) sp.4; 37: N. (Prosthodocis) sp.5; 38: N. (Prosthodocis) sp. (Escala: 0.5mm).

Fig. 30-38: Male genitaly: 30: N. (Prosthodocis) sp.2; 31: N. (Prosthodocis) sp.; 32: N. (Prosthodocis) sp.7; 33: P. (Phytodietus) moragai; 34: N. (Prosthodocis) sp.6; 35: N. (Prosthodocis) sp.12; 36: N. (Prosthodocis) sp.4; 37: N. (Prosthodocis) sp.5; 38: N. (Prosthodocis) sp. (Scale: $0.5 \mathrm{~mm}$ ). 

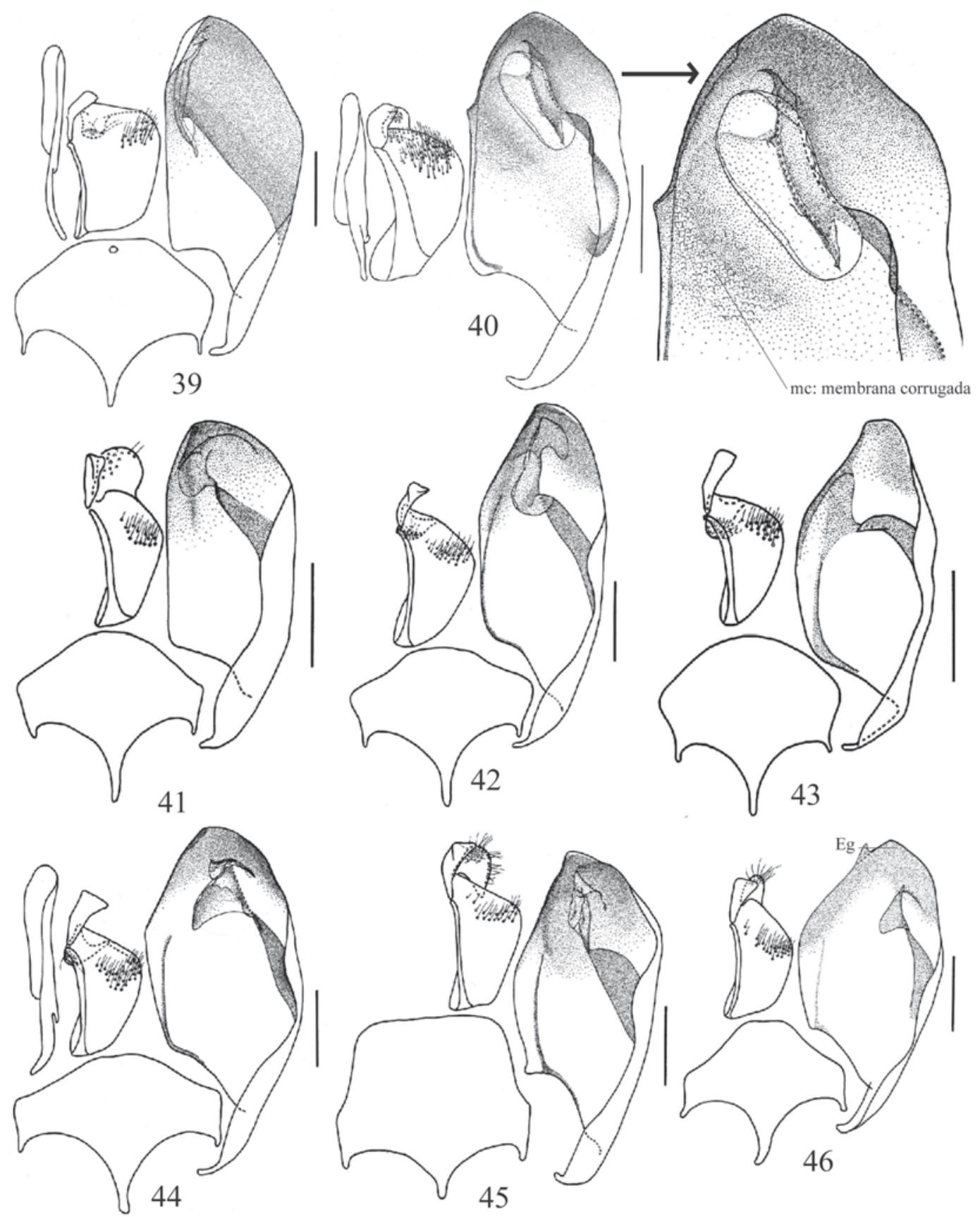

Fig. 39-46: Genitales de machos: 39: N. (Netelia) sp.29; 40: N. (Bessobates) sp.19; 41: N. (Netelia) sp.24; 42: N. (Netelia) sp.34; 43: N. (Netelia) sp.; 44: N. (Netelia) sp.51; 45: N. (Netelia) ignota; 46: N. (Netelia) sp.36 (Escala: 0.5mm).

Fig. 39-46: Male genitaly: 39: N. (Netelia) sp.29; 40: N. (Bessobates) sp.19; 41: N. (Netelia) sp.24; 42: N. (Netelia) sp.34; 43: N. (Netelia) sp.; 44: N. (Netelia) sp.51; 45: N. (Netelia) ignota; 46: N. (Netelia) sp.36 (Scale: 0.5mm). 

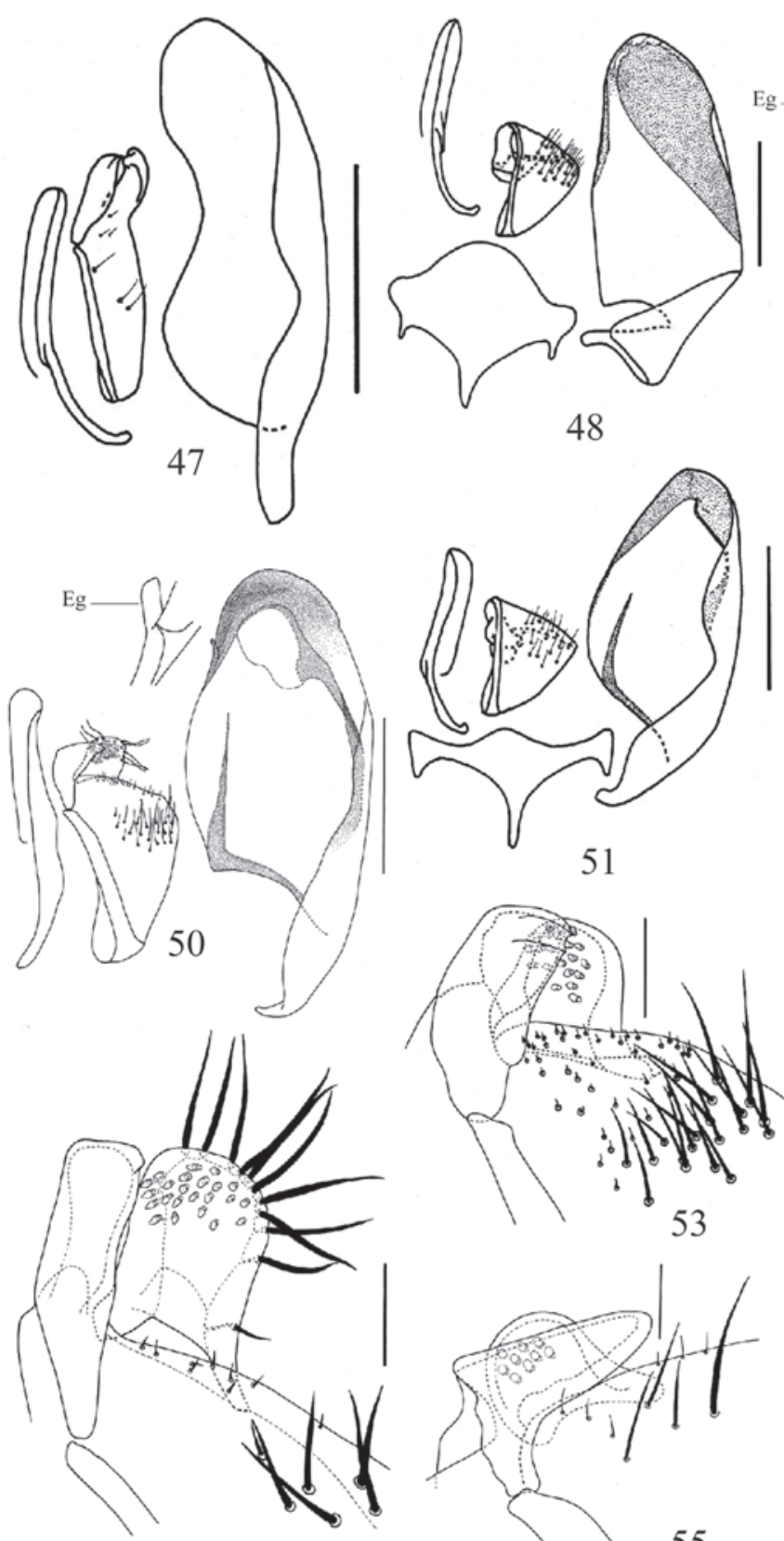

54
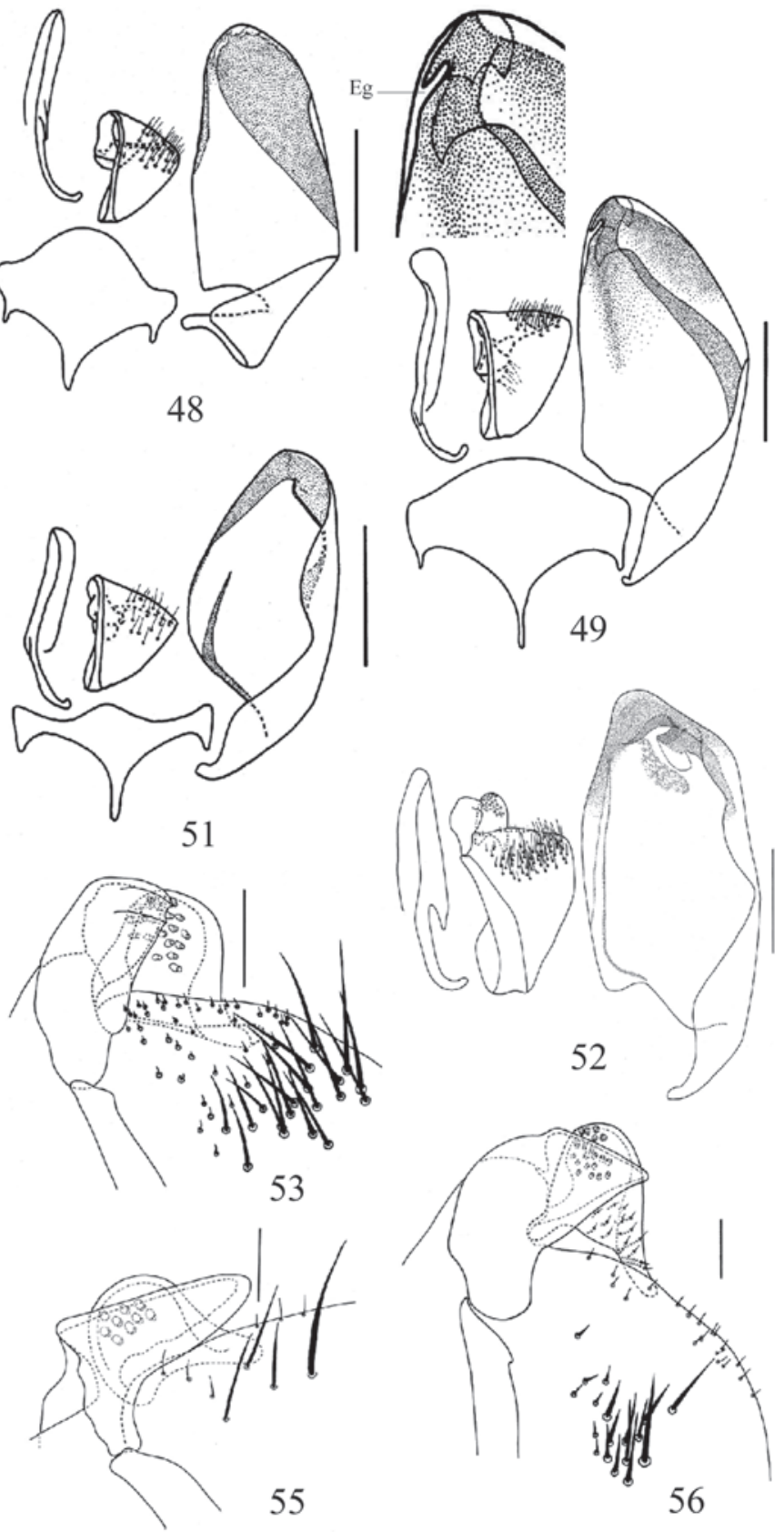

56

Fig. 47-56: Genitales de machos: 47: N. (Monomacrodon) bicolor; 48: N. (Prosthodocis) excerta; 49: N. (Prosthodocis) antefurcalis; 50: $N$. (Apatagium) pagoni; 51: $N$. (Apatagium) $n r$. retaki; 52: N. (Bessobates) virgata; 53: $N$. (Bessobates) sp.19; 54: $N$. (Netelia) sp; 55: $N$. (Parabates) nigricarpus; 56: $N$. (Prosthodocis) sp.5 (Escala: Figs: 47-51=0.5mm; Figs: $53-56=0.1 \mathrm{~mm})$.

Fig. 47-56: Male genitaly: 47: N. (Monomacrodon) bicolor; 48: N. (Prosthodocis) excerta; 49: N. (Prosthodocis) antefurcalis; 50: N. (Apatagium) pagoni; 51: N. (Apatagium) $n r$. retaki; 52: N. (Bessobates) virgata; 53: N. (Bessobates) sp.19; 54: $N$. (Netelia) sp; 55: $N$. (Parabates) nigricarpus; 56: N. (Prosthodocis) sp.5 (Scale: Figs: 47-51=0.5mm; Figs: 53-56=0.1mm). 

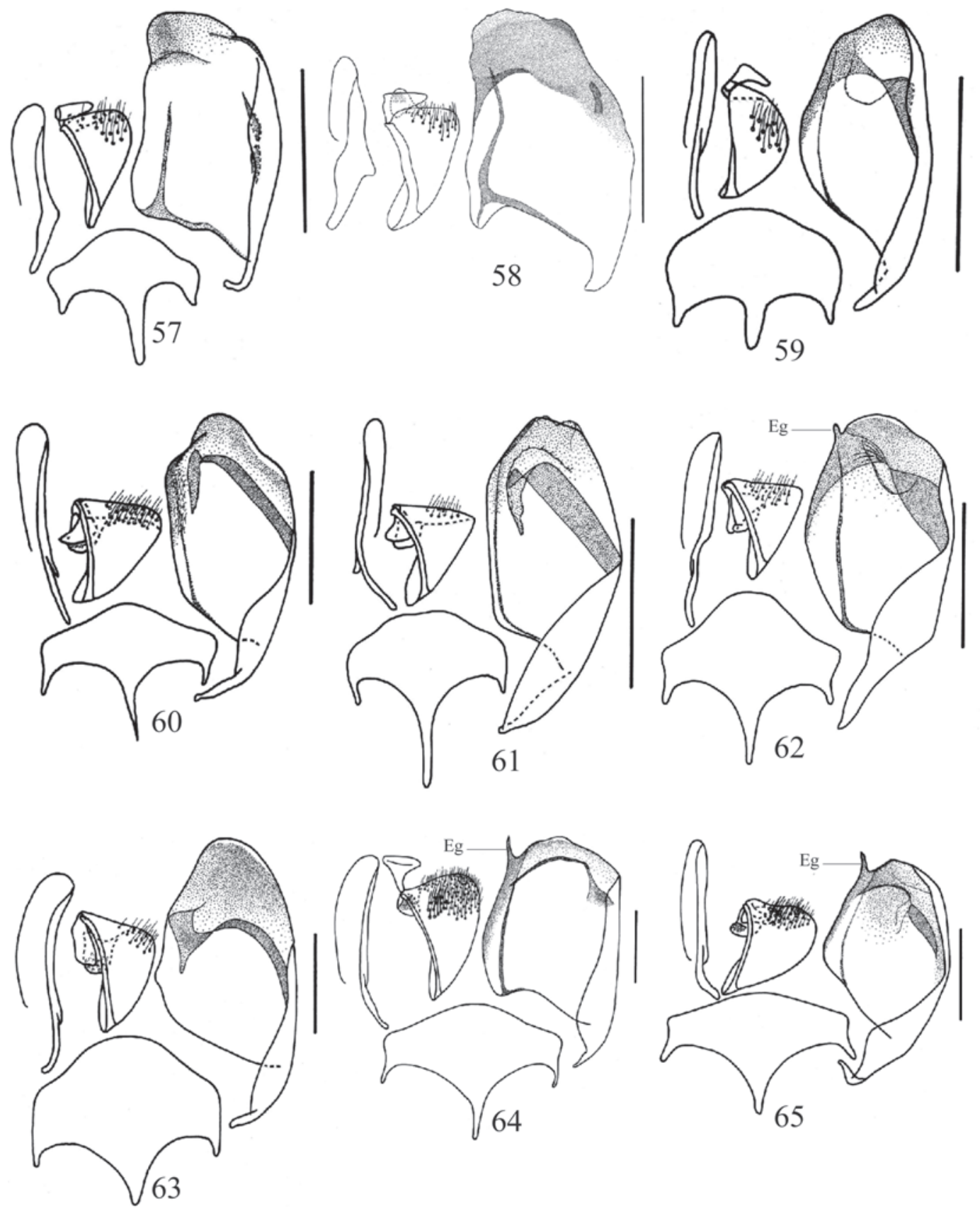

Fig. 57-65: Genitales de machos: 57: N. (Parabates) johnsoni; 58: N. (Parabates) nigricarpus; 59: N. (Longiterebates) himalayensis; 60: N. (Longiterebates) turgida; 61: N. (Paropheltes) parvula; 62: N. (Paropheltes) barberi; 63: N. (Toxochiloides) latro; 64: N. (Toxochilus) magniceps; 65: N. (Toxochilus) clypeata (Escala: 0.5mm).

Fig. 57-65: Male genitaly: 57: N. (Parabates) johnsoni; 58: N. (Parabates) nigricarpus; 59: N. (Longiterebates) himalayensis; 60: N. (Longiterebates) turgida; 61: N. (Paropheltes) parvula; 62: N. (Paropheltes) barberi; 63: N. (Toxochiloides) latro; 64: N. (Toxochilus) magniceps; 65: $N$. (Toxochilus) clypeata (Scale: $0.5 \mathrm{~mm}$ ). 
margen ventral y finaliza en el centro o en el tercio apical de la gonoescama (Fig. 40-46, 49-52, 57-65).

124. Tirante muy esclerosado, generalmente transversal, a veces torcido sobre su largo (Fig. 43).

125. Tirante vestigial (Fig. 57-58).

126. Almohadilla de forma laminar, a veces muy reducida e inconspicua (Fig. 40-46, $50,52)$.

127. Almohadilla membranosa no laminar, a veces en forma de vejiga (Fig. 34, 37).

128. Almohadilla laminar, el tirante se une a ella por debajo de su superficie (Fig. 40).

129. Superficie interna de la gonoescama con una pequeña membrana corrugada cerca a su margen ventral (Fig. 40).

130. Basivolsella amplia, su ancho 0.4-0.8x el largo de su tirante (compare las Fig. 33 y 47 con el resto de figuras de los órganos genitales de los machos).

131. Basivolsella amplia en forma de "media luna" (Fig. 31-32).

132. Basivolsella amplia en forma "triangular" (Fig. 50).

133. Pelos largos en la basivolsella agrupados (compare las Fig. 33 y 47 con el resto de figuras de los órganos genitales de los machos).

134. Basivolsella con un moderado número de pelos largos: 25-40.

135. Basivolsella con un elevado número de pelos largos: 45 o más.

136. Basivolsella con algunos pelos largos mucho más gruesos que los demás (Fig. 64-65).

137. Pelos largos marginales en la distiovolsella ausentes (Fig. 53, 55-56, compare con la Fig. 54).

138. Distiovolsella con muchos pelos largos marginales: 10 o más.

139. Pelos cortos en la superficie ventral de la distiovolsella, ubicados hacia la esquina ventral-basal, presentes (Fig. 56).

140. Distiovolsella con un número moderado de papilas (20-30) en su superficie interna.
141. Distiovolsella con un número elevado de papilas (más de 30) en su superficie interna.

142. Gonolacinia con el margen distal muy amplio y la esquina ventral aguda (Fig. $50)$.

143. Gonolacinia con el margen distal muy amplio y las esquinas ventral y dorsal agudas (Fig. 64).

144. Gonolacinia muy corta, con el margen distal muy amplio y la esquina ventral aguda (Fig. 55).

145. Gonolacinia con el margen distal angosto y con un par de pliegues conspicuos sobre la superficie ventral, el pliegue distal se extiende sobre el margen apical y finaliza sobre la esquina ventral a manera de "diente" (Fig. 53).

146. Gonolacinia ligeramente torcida, con el margen distal amplio y la esquina ventral aguda (Fig. 56).

147. Aedeago muy corto con la mitad basal muy ancha y el margen dorsal convexo (Fig. 57-58).

148. Aedeago corto, su largo 1.0-0.5x el largo de la gonoescama.

149. Plato subgenital con un pequeño orificio cerca de su margen distal (Fig. 39).

Análisis: Para analizar la matriz de taxones vs. caracteres del Apéndice 1 se utilizaron los programas Hennig86 1.5 de James Farris 1988 en conjunto con Tree Gardener 2.2 de Tiago Courrol-Ramos. Se utilizaron dos estrategias de búsqueda para encontrar a los árboles más parsimoniosos, sin y con ponderación de los caracteres (peso sucesivo), a través de los requerimientos programados de origen en los comandos mhennig* y bb*. Los resultados se representan gráficamente para cada análisis a través de un árbol consenso Majority rule.

\section{RESULTADOS}

Todos los resultados se encuentran resumidos en el Cuadro 1 y las Fig. 66-67. 
CUADRO 1

Respaldo filogenético encontrado a través de las dos estrategias de búsqueda utilizadas para hallar a los árboles más parsimoniosos

TABLE 1

Phylogenetic support found through the two search strategies used to find to the most parsimonious trees

\begin{tabular}{|c|c|c|c|c|c|}
\hline & sps & cps & & sps & cps \\
\hline Phytodietini & + & + & Pa. parvula $+N .($ Toxochilus $)$ & + & + \\
\hline Phytodietus & + & + & $*+P a$. sp. 14 & + & + \\
\hline Netelia & + & + & $*+N e . s p .51-53$ & - & + \\
\hline N. (Apatagium) & + & + & N. (Prosthodocis) & - & - \\
\hline N. (Monomacrodon) & + & + & Pr. sp.3-13 + Pr. exserta & + & + \\
\hline$N .($ Apatagium $)+N .($ Monomacrodon $)$ & - & - & $* *+\operatorname{Pr}$ antefurcalis & + & - \\
\hline N. (Bessobates) & + & + & Pr. sp.1, 3-13 + N. (Longiterebates) & - & - \\
\hline grupo virgata & - & - & N. (Netelia $)$ & - & - \\
\hline N. (Parabates $)$ & + & + & grupo geminata & - & - \\
\hline N. (Bessobates $)+N$. (Parabates $)$ & + & + & grupo leo & + & + \\
\hline N. (Longiterebates) & + & - & grupo unguicularis & + & + \\
\hline N. (Protonetelia $)$ & + & + & grupo emorsa & + & + \\
\hline N. (Toxochilus) & + & + & $N .($ Toxochiloides $)$ [= grupo emorsa $]$ & + & + \\
\hline N. (Paropheltes) & - & - & N. (Toxochiloides) latro + Ne. sp.31-32 & + & + \\
\hline
\end{tabular}

$\mathbf{s p s}=$ análisis sin peso sucesivo, $\mathbf{c p s}=$ análisis con peso sucesivo, Ne. $=N .($ Netelia $), \boldsymbol{P a} .=N .($ Paropheltes $), \boldsymbol{P r}=N$. (Prosthodocis), += el análisis respalda la monofilia del grupo, $-=$ el análisis no respalda la monofilia del grupo, *= grupo conformado por Pa. parvula $+N$. (Toxochilus), **= grupo conformado por Pr. sp.3-13+Pr. exserta.

$\mathbf{s p s}=$ analysis without successive weighting, $\mathbf{c p s}=$ analysis with successive weighting, Ne. $=$ N. $($ Netelia $), \boldsymbol{P a} .=N$. (Paropheltes), $\boldsymbol{P r} .=N$. (Prosthodocis),$+=$ the analysis supports the monophyly of the group, $-=$ the analysis does not support the monophyly of the group, $*=$ group conformed by Pa. parvula $+N$. (Toxochilus), $* *=$ group conformed by $P r$. sp. $3-13$ + Pr. exserta.

Las dos estrategias de búsqueda utilizadas para hallar a los árboles más parsimoniosos sustentan las monofilias de la tribu Phytodietini y la de los géneros Phytodietus y Netelia.

Seis de los 11 subgéneros de Netelia recibieron respaldo como grupos monofiléticos o como linajes monotípicos filogenéticamente independientes en ambas estrategias de búsqueda: N. (Apatagium), N. (Bessobates), N. (Monomacrodon), N. (Parabates), N. (Protonetelia) y $N$. (Toxochilus). De igual forma obtuvieron soporte las monofilias de los grupos de especies leo, unguicularis y emorsa propuestos por Townes (1938) para el subgénero N. (Netelia) en la región Neártica.

La monofilia de $N$. (Longiterebates) sólo encontró apoyo en el análisis que no involucra la ponderación de los caracteres (Fig. 66).
La especie $N$. (Toxochiloides) latro en ambos análisis conformó un grupo monofilético con las especies $N$. (Netelia) sp. 31 y 32, representantes del grupo emorsa Townes.

Las especies $N$. (Netelia) sp. 51-53 sólo conformaron un grupo monofilético con el grupo N. (Toxochilus) $+N$. (Paropheltes) parvula en el análisis con peso sucesivo (Fig. 67).

No se encontró soporte para las monofilias de los subgéneros N. (Netelia), N. (Paropheltes) y N. (Prosthodocis); tampoco para los grupos de especies virgata de $N$. (Bessobates) y geminata Townes de N. (Netelia).

Las especies determinadas aquí como $N$. (Prosthodocis) conformaron tres (Fig. 66) o cuatro (Fig. 67) grupos en los cladogramas resultantes. Aunque un grupo representativo de especies (spp. 3-13) conformó un grupo 


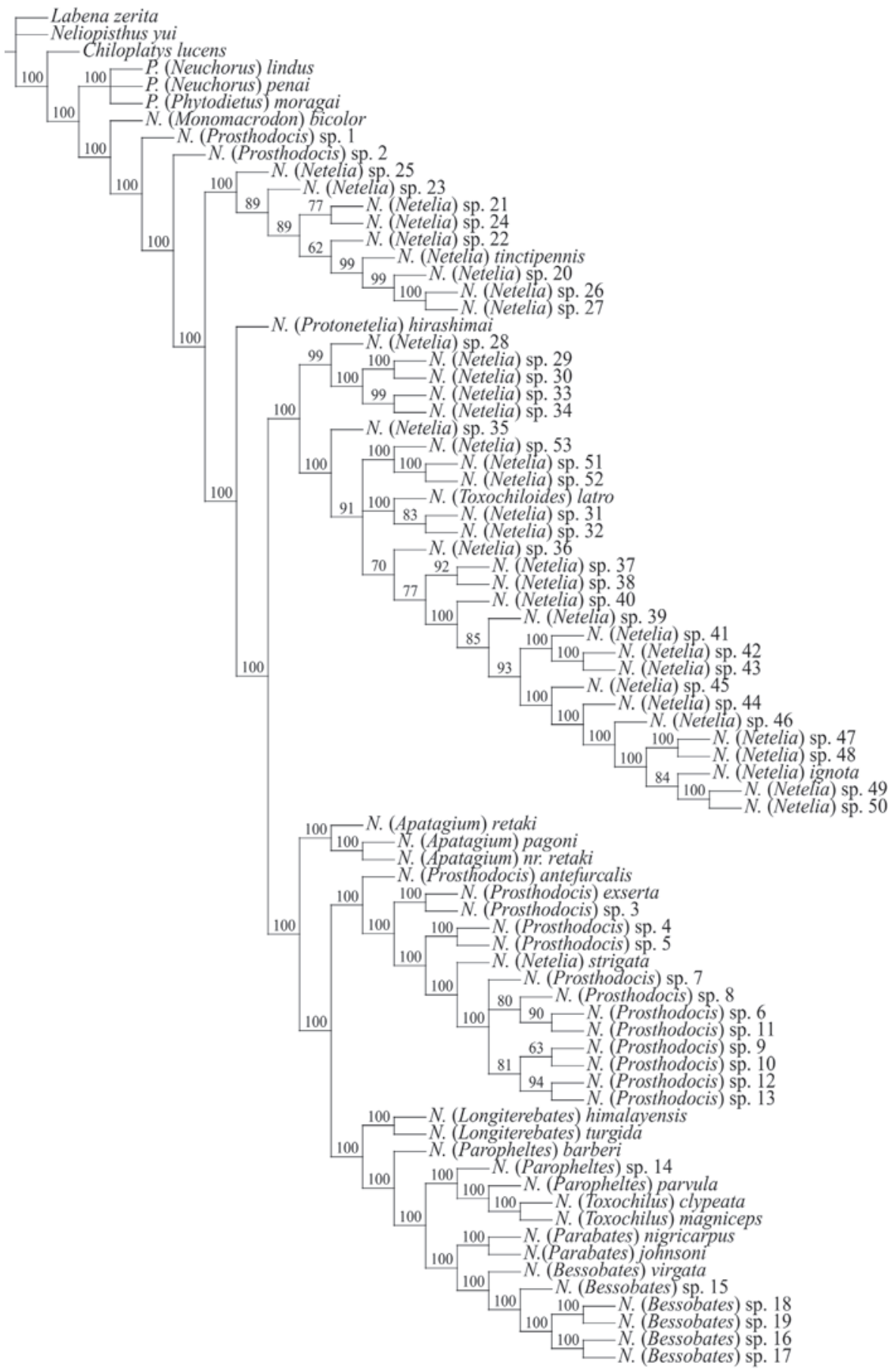

Fig. 66. Consenso Majority rule (largo $=649 ; \mathrm{Ci}=23 ; \mathrm{Ri}=68$ ) obtenido a partir de 1211 árboles (largo=649; $\mathrm{Ci}=23 ; \mathrm{Ri}=$ 68) resultado del análisis $b b^{*}$ mhennig* $\sin$ peso sucesivo.

Fig. 66. Majority rule consensus (long=649; $\mathrm{Ci}=23 ; \mathrm{Ri}=68$ ) obtained through 1211 trees (long=649; $\mathrm{Ci}=23 ; \mathrm{Ri}=68$ ) result of the analysis $b b *$ mhennig * without successive weighting. 


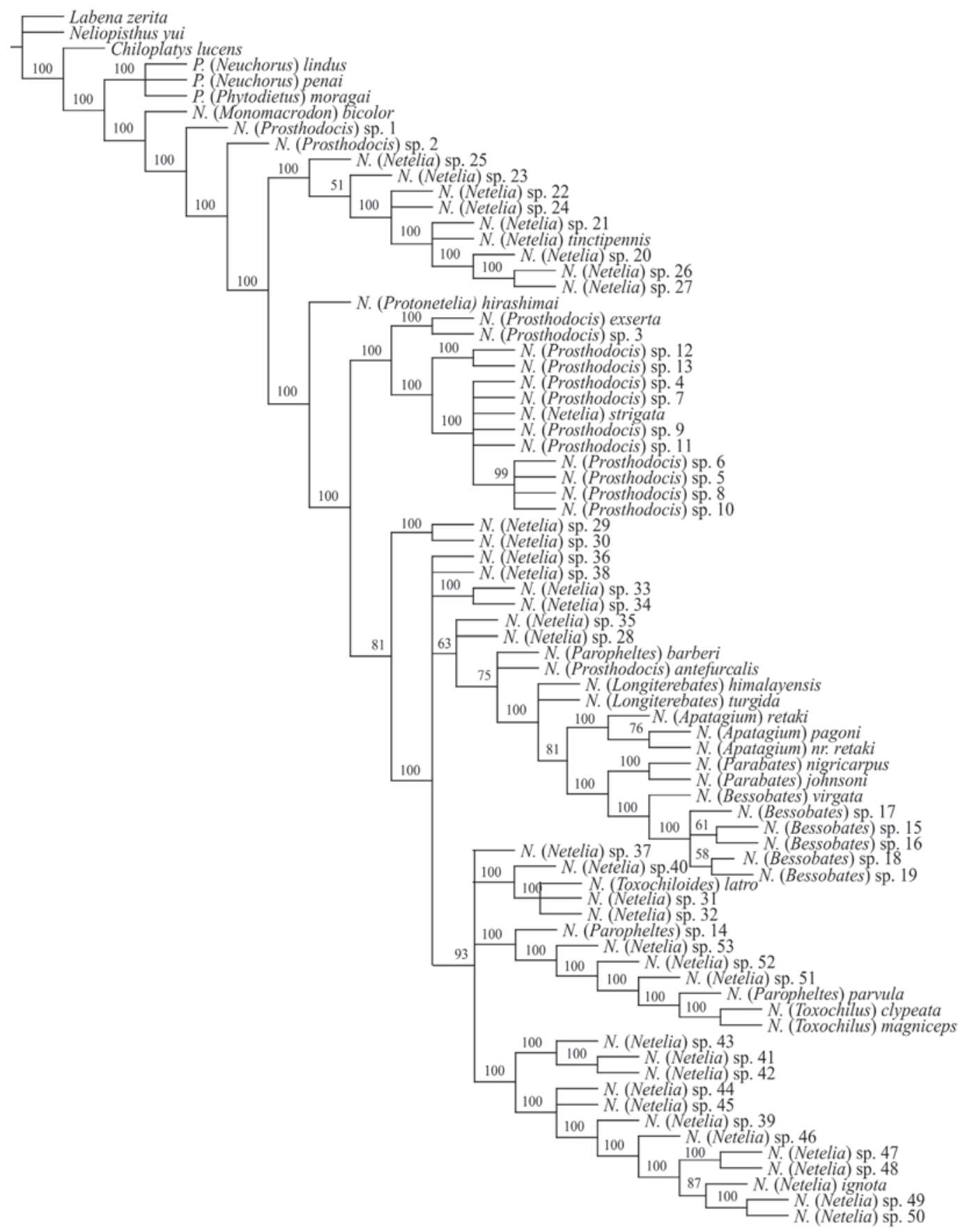

Fig. 67. Consenso Majority rule ( $\operatorname{largo}=721 ; \mathrm{Ci}=20 ; \mathrm{Ri}=64)$ obtenido a partir de 1211 árboles $(\operatorname{largo}=719 ; \mathrm{Ci}=20 ; \mathrm{Ri}=$ 64) resultado del análisis $b b^{*}$ mhennig* con peso sucesivo.

Fig. 67. Majority rule consensus (long= 721; $\mathrm{Ci}=20 ; \mathrm{Ri}=64$ ) obtained through 1211 trees (long=719; $\mathrm{Ci}=20 ; \mathrm{Ri}=64$ ) result of the analysis $b b *$ mhennig * with successive weighting. 
monofilético con $N$. (Pr.) antefurcalis $+N$. (Pr.) exserta en el análisis sin peso sucesivo (Fig. 66), y sólo con N. (Pr.) exserta en el análisis con peso sucesivo (Fig. 67).

Las tres especies examinadas de $N$. (Paropheltes) nunca conformaron un grupo monofilético, y los resultados de ambos análisis indican que N. (Pa.) parvula y N. (Paropheltes) sp. 14 parecen estar más relacionadas filogenéticamente con las especies de $N$. (Toxochilus).

Las especies determinadas aquí como $N$. (Netelia) conformaron dos (Fig. 66) o múltiples (Fig. 67) grupos en los cladogramas resultantes.

Las especies $N$. (Monomacrodon) bicolor, N. (Prosthodocis) sp. 1 y N. (Prosthodocis) sp. 2 en ambos análisis ocuparon las posiciones más basales en la filogenia del género Netelia. Mientras que N. (Mo.) bicolor nunca conformó un grupo monofilético con las tres especies examinadas de $N$. (Apatagium).

En ninguno de los dos análisis las especies costarricenses de Netelia, principalmente las determinadas como N. (Prosthodocis), conformaron algún grupo monofilético con las especies representantes de los subgéneros $N$. (Parabates) o $N$. (Longiterebates). Sin embargo, como grupos independientes, las especies costarricenses de N. (Bessobates) y las examinadas de $N$. (Parabates) si demostraron, en ambos análisis, que los dos subgéneros parecen estar filogenéticamente relacionados.

\section{DISCUSIÓN}

La monofilia de Netelia da sustento a la hipótesis de Townes (1938) de agrupar a todos los subgéneros (antes de Townes considerados géneros) en una unidad morfológicamente homogénea, que permita su fácil identificación como género.

Cuando Townes (1938) definió a N. (Prosthodocis) lo hizo utilizando para ello a la especie Tipo del subgénero $N$. (Pr.) antefurcalis Szépligeti de África y a N. (Pr.) exserta Cushman de Norte América. Él describió la morfología externa del subgénero basándose en las dos especies, pero la de los órganos genitales de los machos sólo con la morfología de exserta (Fig. 48). Durante la presente investigación se revisaron los genitales de los machos de ambas especies, y se pudo constatar que los de antefurcalis (Fig. 49) tienen más en común con los genitales de otros subgéneros que con la descripción realizada por Townes para N. (Prosthodocis). Por ejemplo, las gonoescamas de los genitales de los machos de $N$. (Prosthodocis) sensu Townes tienen la membrana interna modificada en un tirante amplio y poco conspicuo o carecen de tal transformación (Fig. 30-32, 34-38, 48), mientras que la de $N$. (Pr.) antefurcalis tienen un tirante conspicuo, angosto y oblicuo, similar a como lo presentan las especies de los subgéneros $N$. (Apatagium), N. (Bessobates), N. (Netelia), N. (Toxochiloides), N. (Longiterebates), N. (Paropheltes) y N. (Toxochilus) (Fig. 40-46, 50-51, 59-65). De esta manera, es muy probable que a lo que se le llama N. (Prosthodocis) en el mundo (Kaur \& Jonathan 1979, Konishi 1991, 1992, Kasparyan \& Tolkanitz 1999), siguiendo el concepto de Townes (1938), sean en realidad otros taxones no relacionados filogenéticamente con $N$. (Pr.) antefurcalis. Las especies costarricenses que se logran determinar como $N$. (Prosthodocis) s.T. representan a tres grupos filogenéticamente independientes entre ellos, y dos de estos claramente independientes de $N$. (Pr.) antefurcalis. Es muy probable que los tres grupos de especies presentes en Costa Rica representen a nuevos subgéneros de Netelia.

N. (Paropheltes) está compuesto en el mundo por siete grupos de especies: flavolineata, thomsoni, guptai, ciliata, barberi, tarsata y radiata (Townes 1938, Kaur \& Jonathan 1979, Konishi 1996a). En la presente investigación se revisaron especies representantes de los tres últimos grupos. Sumado al hecho que estas especies nunca conformaron un grupo monofilético, algunos de los caracteres que se utilizan en la literatura para definir al subgénero han sido reconocidos en la presente investigación como plesiomorfías y otros resultaron ser convergentes con otros subgéneros. Además, aunque no se tuvo la oportunidad de revisar especies de todos los grupos, al interpretar las 
definiciones de éstos en la literatura (Townes 1938, Kaur \& Jonathan 1979, Konishi 1996a), se nota una gran heterogeneidad morfológica, lo que contribuye a pensar que el subgénero es un conjunto completamente artificial de especies. Este asunto se complica más al considerar que la especie Tipo para el subgénero, $N$. (Pa.) flavolineata Cameron (Townes 1938), es documentada sólo para la India y es conocida sólo por la hembra, por lo que se ignora la morfología de los genitales de los machos; además, según Kaur \& Jonathan (1979) el ovipositor relativamente corto y las patas relativamente gruesas sugieren alguna relación con $N$. (Bessobates). Ambos caracteres son reconocidos aquí como derivados, y el primero como sinapomórfico para $N$. (Bessobates). Siguiendo los conceptos modernos en sistemática filogenética, es muy probable que algunos de los grupos de especies de $N$. (Paropheltes), o tal vez todos, en el futuro deban ser elevados a la categoría taxonómica de subgéneros, correspondiéndole el nombre de $N$. (Paropheltes) al grupo flavolineata, restringido a la región Oriental.

$N$. (Netelia) es el subgénero mayor, en éste se han clasificado a más del $70 \%$ de las especies descritas de Netelia en el mundo (Yu \& Horstmann 1997). Townes (1938) reconoció para el Neártico a cinco grupos de especies: unguicularis, emorsa, leo, geminata e idioctenus. Aquí se incluyeron especies de los cuatro primeros. Igual como sucede con $N$. (Netelia), en relación con los demás subgéneros, en el grupo geminata se conjuntan a todas las especies que no calzan con las definiciones propuestas para los demás grupos de especies. Por esta razón, no es sorpresa la falta de una relación monofilética entre las especies costarricenses que se determinaron como $N$. (Netelia) y grupo geminata, y apoya la idea de Konishi (2005) de la falta de naturalidad de este subgénero.

En las revisiones post-Townes que se realizaron para Netelia en otras partes del mundo, no se clasificaron a las especies de $N$. (Netelia) en los grupos propuestos por Townes (1938). Sin embargo, el subgénero post-Townes $N$. (Toxochiloides), documentado hasta antes de esta investigación para las regiones Paleártica y
Oriental (Yu \& Horstmann 1997), corresponde, según los resultados obtenidos aquí, al grupo emorsa Townes del Nuevo Mundo, una idea que ya había sido sugerida por Konishi (1996b) en su revisión del subgénero para el Japón.

Debido a la falta de naturalidad de $N$. (Netelia) y a que algunos de los grupos que lo componen si son monofiléticos [por ejemplo, unguicularis, leo y emorsa -ahora $N$. (Toxochiloides)-], es muy probable que en el futuro estos grupos deban ser clasificados en la categoría taxonómica de subgéneros.

Gauld (1997) informó que N. (Bessobates), N. (Netelia), N. (Prosthodocis), N. (Parabates) y $N$. (Longiterebates) son los subgéneros de Netelia de posible presencia en Costa Rica. Sin embargo, la presente investigación confirma la presencia incondicional de $N$. (Bessobates) y con fines prácticos las de $N$. (Netelia) y $N$. (Prosthodocis); también descarta sin lugar a dudas la presencia de $N$. (Parabates) y $N$. (Longiterebates); y además sugiere la presencia incondicional de $N$. (Toxochiloides) y tentativamente la de N. (Paropheltes) y N. (Toxochilus), este último en un sentido más amplio al definido por Townes (1938).

N. (Longiterebates) y N. (Parabates) son grupos monofiléticos, relativamente fáciles de determinar, ninguna de las especies de Netelia hasta ahora recolectadas en Costa Rica cumple satisfactoriamente con la combinación de atributos que caracterizan a ambos subgéneros, ni las especies incluidas en el presente estudio conformaron ningún grupo monofilético con ellos. De esta manera, $N$. (Longiterebates) debe mantenerse como un subgénero restringido a la Cordillera del Himalaya en la región biogeográfica Oriental (Kaur \& Jonathan 1979, Yu \& Horstmann 1997); mientras que N. (Parabates) en el Nuevo Mundo debe quedar restringido al Neártico (Townes 1938, Yu \& Horstmann 1997). El hecho de que $N$. (Parabates) tiene especies presentes en América del Norte deja cierto margen de probabilidad para que especies de este subgénero sean documentadas en el futuro para alguna localidad neotropical, inclusive Costa Rica; algo más difícil que ocurra con N. (Logiterebates). 
N. (Bessobates) y N. (Toxochiloides) son subgéneros monofiléticos, claramente definidos y muy fáciles de reconocer. No cabe duda que las especies costarricenses que han sido determinadas como parte de estos subgéneros están filogenéticamente relacionadas con especies de ambos en otras latitudes. Mientras que $N$. (Netelia), a pesar de no ser monofilético, debe mantenerse para clasificar a las especies costarricenses que no puedan ser ubicadas en los demás subgéneros. Por otro lado, aunque el concepto de N. (Prosthodocis) es muy confuso, es mejor considerar a las especies costarricenses como parte de este subgénero hasta tener más estudios filogenéticos.

El registro sobre la presencia en Costa Rica de los subgéneros $N$. (Paropheltes) y $N$. (Toxochilus) es temporal, principalmente para el último subgénero, hasta contar con más estudios filogenéticos. La especie $N$. (Pa.) sp. 14 se determina muy bien como $N$. (Paropheltes), pero tanto ésta como las especies $N$. (Ne.) sp. 51-53 conformaron un grupo monofilético con las especies examinadas de $N$. (Toxochilus) de Norte América.

La posible relación filogenética entre $N$. (Monomacrodon) y N. (Apatagium) desde Townes (1938) ha sido insinuada, principalmente por Gauld (1983). Esta supuesta relación era sostenida por compartir las especies de ambos subgéneros una modificación en los peines tarsales posteriores, tal como se describe en el carácter 81 (Fig. 16). Los análisis cladísticos efectuados aquí no encontraron apoyo para la relación entre los dos subgéneros, y demostraron que la modificación de las uñas tarsales posteriores es una convergencia para ambos conjuntos de especies. Por otro lado, Townes (1938) postuló que $N$. (Monomacrodon) era posiblemente el subgénero más derivado de Netelia, debido a que su morfología externa era más especializada que la de los demás subgéneros; mientras que la poca especialización que mostraban sus genitales masculinos según él se debía a una reversión hacia la condición más plesiomórfica. Los resultados obtenidos en los análisis cladísticos sugieren todo lo contrario (Fig. 66-67). N. (Monomacrodon) es posiblemente el subgénero más basal en la filogenia de Netelia, y las plesiomorfías encontradas en la morfología de sus genitales no se deben a reversiones evolutivas. Además, las especializaciones en su morfología externa son productos de convergencias, como en el carácter antes mencionado, o a autopomorfías surgidas en este subgénero, como es el caso del esculpido de su clípeo, mesosoma y propodeo.

El presente estudio encontró evidencias que sugieren que el género Netelia y los subgéneros N. (Apatagium), N. (Bessobates), $N$. (Longiterebates), N. (Monomacrodon), N. (Parabates), N. (Protonetelia), N. (Toxochilus) y $N$. (Toxochiloides) son grupos monofiléticos; mientras que $N$. (Netelia), N. (Paropheltes) y N. (Prosthodocis) son probablemente grupos polifiléticos. En el futuro otros estudios tendrán la responsabilidad de probar estas hipótesis analizando más caracteres morfológicos y moleculares, utilizando nuevas estrategias de búsquedas de los árboles más parsimoniosos o estadísticamente más probables, e incorporando a la matriz más especies de cada subgénero, principalmente de $N$. (Netelia), N. (Paropheltes) y N. (Prosthodocis), provenientes de localidades que cubran una mayor distribución mundial.

Los subgéneros de Netelia, principalmente los no monofiléticos, necesitan de una redefinición taxonómica más acorde con los conceptos modernos en sistemática filogenética. Igual que en muchos otros himenópteros (Gauld \& Mound 1982), la morfología de Netelia es altamente homoplásica, lo que ha contribuido a delimitar artificialmente a los subgéneros. Es recomendable que $N$. (Netelia), $N$. (Paropheltes) y $N$. (Prosthodocis) sean subdivididos en múltiples subgéneros, aumentando considerablemente el número de éstos en el futuro. Los siete grupos de especies reconocidos en el mundo de $N$. (Paropheltes) deben ser elevados a la categoría de subgéneros. Mientras que sólo las especies costarricenses que logran determinarse como N. (Prosthodocis) y $N$. (Netelia) probablemente representen a tres $\mathrm{y}$ más de siete subgéneros, respectivamente, si se siguen estrictamente las reglas en sistemática 
filogenética para reconocer únicamente a grupos naturales. Para un género de insectos tan diverso como Netelia, con una distribución mundial que abarca casi a todas las regiones biogeográficas, no es sorprendente que sus especies requieran clasificarse en un elevado número de subgéneros.

Con los resultados de este estudio podemos establecer que la distribución mundial de todos los subgéneros, de la manera como son taxonómicamente definidos en la actualidad, es la que se muestra en el Apéndice 2. Comprobándose la presencia en el Neotrópico de los subgéneros N. (Bessobates), N. (Netelia), N. (Prosthodocis) y N. (Paropheltes), los tres primeros documentados por Gauld (1997) y Townes \& Townes (1966) para Costa Rica y otras localidades tropicales del Nuevo Mundo, respectivamente; la existencia del último había sido sugerida por Townes (1938) cuando mencionó el depósito de una hembra en la colección de la Universidad de Cornell, recolectada en Perú, reconocida por él como una especie no descrita de $N$. (Paropheltes). Para los subgéneros N. (Toxochilus) y N. (Toxochiloides) es la primera vez que se registra su existencia en el Neotrópico, aunque anteriormente la presencia de $N$. (Toxochiloides) fue documentada a través del grupo emorsa de $N$. (Netelia) por Townes (1938), quién había mencionado la gran diversidad de este grupo de especies en el Neotrópico.

En el Apéndice 3 se ofrece una clave para determinar a los subgéneros presentes en Costa Rica, la cual también puede ser de gran ayuda para identificar provisionalmente a los subgéneros existentes en otros sitios neotropicales. Esta clave es una mejora a la elaborada por Gauld (1997). En ella se excluyeron a los subgéneros $N$. (Parabates) y N. (Longiterebates), equívocamente documentados por Gauld para Costa Rica, y se incorporan a los subgéneros no mencionados por el mismo autor N. (Paropheltes), N. (Toxochilus) y N. (Toxochiloides).

\section{AGRADECIMIENTOS}

A la Oficina Regional Centroamericana de la Agencia Alemana de Intercambio
Académico (DAAD), San José, Costa Rica; a la Organización para Estudios Tropicales de Costa Rica (OET); al Instituto Smithsonian de Investigaciones Tropicales de Panamá (STRI); y al Instituto Nacional de Biodiversidad de Costa Rica (INBio) por financiarme los estudios de Maestría y parte de los costos de investigación, respectivamente. Al Sistema de Estudios de Posgrado en Biología de la Universidad de Costa Rica. A David Wahl del American Entomological Institute (AEI), Gainesville-Florida-EEUU; Andrew Bennett del Royal Ontario Museum, Ontario-Canada; e Ian Gauld (q.e.p.d.) del Natural History Museum (NHM) de Londres-Inglaterra, por el préstamo de especímenes representantes de diferentes subgéneros de Netelia provenientes de diferentes regiones del mundo. A la Biblioteca del STRI por la facilitación de literatura. Al Museo de Insectos (Escuela de Agronomía) y al Laboratorio de Entomología (Escuela de Biología) de la UCR por facilitar espacio y equipo durante el desarrollo de la investigación. A D. Wahl, A. Bennett y James Carpenter por sus orientaciones para el desarrollo de los análisis cladísticos preliminares. A las siguientes personas por sus recomendaciones durante el desarrollo del proyecto de investigación y para mejorar los borradores iniciales del presente documento: Paul Hanson, I. Gauld, Axel Retana y William Eberhard.

\section{RESUMEN}

Se presentan los resultados del primer análisis cladístico realizado para el género Netelia. Éste se llevó a cabo con la ayuda de los programas Hennig86 y Tree Gardener 2.2, a través de una matriz de 79 taxones y 149 caracteres, descritos principalmente de la morfología externa y de los genitales de los machos, sólo tres caracteres están relacionados con la historia natural de las especies. Dentro del grupo de estudio se incluyeron a especies representantes de los 11 subgéneros de Netelia descritos en el mundo, aunque casi el $80 \%$ de las especies examinadas son costarricenses. Para poner a prueba la monofilia del género, también se incluyeron dentro del grupo de estudio a las cinco especies trifoninas Neliopisthus yui, Chiloplatys lucens, Phytodietus (Neuchorus) penai, P. (N.) lindus y P. (Phytodietus) moragai. Se utilizó como especie externa a Labena zerita (Labeninae). Las estrategias de búsqueda de los árboles incluyeron la reconstrucción filogenética 
por parsimonia, sin y con peso sucesivo de los caracteres, a través de los comandos bb* y mhennig*. En el análisis sin peso sucesivo se obtuvieron 1211 árboles igualmente parsimoniosos con un largo de 649 pasos, un $C i$ de 23 y un $R i$ de 68; mientras que en el análisis con peso sucesivo se obtuvieron 1211 árboles con un largo de 719 pasos, un $C i$ de 20 y un $R i$ de 64. Los múltiples cladogramas obtenidos fueron resumidos a través de dos árboles consensos Majority rule, uno para cada estrategia de búsqueda. Se encontró sustento a favor de la monofilia del género Netelia, y los subgéneros N. (Apatagium), N. (Bessobates), N. (Monomacrodon), N. (Parabates), N. (Protonetelia), N. (Toxochilus) y N. (Longiterebates), aunque este último resultó monofilético sólo en el análisis sin peso sucesivo. También se obtuvo soporte filogenético para los grupos de especies leo, unguicularis y emorsa propuestos por Townes (1938) para el subgénero $N$. (Netelia). Los resultados respaldan la sinonimia de $N$. (Toxochiloides) con el grupo emorsa del Nuevo Mundo. No se encontró soporte filogenético para los subgéneros N. (Netelia), N. (Prosthodocis) y N. (Paropheltes), ni para los grupos de especies virgata de $N$. (Bessobates) y geminata Townes de N. (Netelia). Tampoco se encontró apoyo para la relación filogenética entre los subgéneros N. (Apatagium) y N. (Monomacrodon). Por último, con los resultados obtenidos aquí se descarta la presencia en Costa Rica de los subgéneros $N$. (Longiterebates) y N. (Parabates), tal como fue documentado para el país por Gauld (1997), y se acepta incondicionalmente la presencia de $N$. (Bessobates), y con fines prácticos la de $N$. (Prosthodocis) y N. (Netelia), demás se propone tentativamente la presencia de $N$. (Paropheltes) y N. (Toxochilus).

Palabras claves: filogenia, morfología, genitales de machos, especies parasitoides, Tryphoninae, insectos, Costa Rica.

\section{REFERENCIAS}

Cameron, P. 1907. Description of new genus and some new species of Hymenoptera captured by lieut. col. C.G. Nurse at Deesa, Matheran and Ferozepore. J. Bombay Nat. Hist. Soc. 17: 1001-1012.

Coronado-Rivera, J. 2007. Netelia Gray (Hymenoptera: Ichneumonidae: Tryponinae) de Costa Rica; y anatomía del sistema reproductor de las hembras de Ichneumonidae. Tesis de Maestría. Universidad de Costa Rica, San José, Costa Rica.

Cushman, R.A. 1934. New Ichneumonidae from India and China. India Forest Rec. Delhi 20: 1-8.

Enderlein, G. 1912. Beiträge zur Kenntnis aussereuropäischer Ichneumoniden. I. Paniscinae. Stettin ent. Ztg. 73: $105-144$
Förster, A.1869. Synopsis der Familien und Gattungen der Ichneumonen. Verh. Naturhist. Ver. Rheinl 25: 135-221.

Gámez, R. \& I. Gauld. 1993. Costa Rica: an innovative approach to the study of tropical biodiversity, p. 329336. En J. La Salle \& I. Gauld (eds.). Hymenoptera and Biodiversity. CABI, Wallingford, U.K.

Gauld, I.D. 1983. Netelia species of the subgenera Apatagium Enderlein and Monomacrodon Cushman (Hyemnoptera: Ichneumonidae) of Brunei with a re-assessment of the suparespecific classification. Brunei Mus. J. 5: 123-143.

Gauld, I.D. 1984. An introduction to the Ichneumonidae of Australia. British Museum (Natural History), London, Inglaterra.

Gauld, I.D. 1997. The Ichneumonidae of Costa Rica. Mem. Am. Entomol. Inst. 57: 1-485.

Gauld I.D. \& L.A. Mound 1982. Homoplasy and the delineation of holophyletic genera in some insect groups. Syst. Entomol. 7: 73-86.

Gauld, I.D. \& D.B. Wahl 2000. The Labeninae (Hymenoptera: Ichneumonidae): a study in phylogenetic reconstruction and evolutionary biology. Zool. J. Linn. Soc-Lond. 129: 271-347.

Gray, J.E. 1860. On the hooks on the front edge of the hinde wings of certain Hymenoptera. Ann. Mag. Nat. Hist. 5: 339-342.

Hanson, P.E. \& I.D. Gauld. 1995. The Hymenoptera of Costa Rica. Oxford University, Oxford, Inglaterra.

Kasparyan, D.R. \& V.I. Tolkanitz. 1999. Fauna of Russia and neighbouring countries. Insecta Hymenoptera. Ichneumonidae subfamilies: Tryphoninae, Eucerotinae, Adelognathinae y Townesioninae 3: 1-404.

Kaur, R. \& J.K. Jonathan 1979. Ichneumonologia orientalis. Pt VIII. The tribe Phytodietini from India (Hyemnoptera: Ichneumonidae). Delhi.

Kaur, R. \& J.K. Jonathan 1976. A new tryphonine subgenus Longiterebates from India (Hyemnoptera: Ichneumonidae). Orient. Insects 10: 33-37.

Konishi, K. 1986. A new subgenus and species of the genus Netelia (Hymenoptera; Ichneumonidae) from New Guinea. Kontyû 54: 415-419.

Konishi, K. 1991. A revision of the subgenus Prosthodocis Enderlein of the genus Netelia Gray of Japan (Hymenoptera, Ichneumonidae), I. Jpn. J. Entomol. 59: 775-788. 
Konishi, K. 1992. A revision of the subgenus Prosthodocis Enderlein of the genus Netelia Gray of Japan (Hymenoptera, Ichneumonidae), II. Jpn. J. Entomol. 60: 39-53.

Konishi, K. 1996a. A revision of the subgenus Paropheltes Cameron of the genus Netelia Gray (Hymenoptera, Ichneumonidae) of Japan. Jpn. J. Entomol. 64: 163187.

Konishi, K. 1996b. Study on the subgenus Toxochiloides Tolkanitz of the genus Netelia Gray (Hymenoptera, Ichneumonidae) of Japan. Jpn. J. Entomol. 64: 473481.

Konishi, K. 2005. A preliminary revision of the subgenus Netelia of the genus Netelia from Japan (Hymenoptera; Ichneumonidae; Tryphoninae). Insecta Matsumurana 62: 45-121.

Morley, C. 1913. A Revision of the Ichneumonidae, London, Inglaterra.

Quicke, D.L.J., C. Lopez-Vaamonde \& R. Belshaw 1999. The basal Ichneumonidae (Insecta: Hymenoptera): 28S D2 rDNA considerations of the Brachycyrtinae, Labeninae, Paxylommatinae and Xoridinae. Zool. Scr. 28: 203-210.
Porter, C. 1998. Guía de los géneros de Ichneumonidae en la Región Neantártica del Sur de Sudamérica. Opera Lilloana 42: 1-80.

Short, J.R.T. 1978. The final larval instar of the Ichneumonidae. Mem. Am. Entomol. Inst. 25: 1-508.

Tolkanitz, V.I. 1974. Revision of the genus Netelia (Hyemnoptera: Ichneumonidae) and review of species of the USSR. Zool. Zhurnal. 53: 376-391.

Townes, H. 1938. The nearctic species of Netelia (Paniscus of authors) and revision of the genera of Netelini. Lloydia 1: 168-231.

Townes, H. 1969. The genera Ichneumonidae. Mem. Am. Entomol. Inst. 11: 1-300.

Townes, H. \& M. Townes. 1966. A catalogue and reclassification of Neotropic Ichneumonidae. Mem. Am. Entomol. Inst. 8: 1-367.

Townes, H., M. Townes \& V.K. Gupta. 1961. A catalogue and reclassification of the Indo-Australian Ichneumonidae. Mem. Am. Entomol. Inst. 1: 1-522.

Yu, D. \& K. Horstmann. 1997. Catalogue of world Ichneumonidae (Hymenoptera). Mem. Am. Entomol. Inst. 58: $1-1558$ 